\title{
Computing the equilibrium configuration of epitaxially strained crystalline films
}

\author{
Eric Bonnetier \\ Centre de Mathématiques Appliquées, CNRS UMR 7641 \\ Ecole Polytechnique, 91128 Palaiseau, France \\ eopus@cmapx.polytechnique.fr \\ Antonin Chambolle \\ CEREMADE, CNRS UMR 7534 \\ Université de Paris-Dauphine \\ Place de Lattre de Tassigny, 75775 Paris Cedex 16, France \\ antonin.chambolle@ceremade.dauphine.fr
}

\begin{abstract}
We study a model for shape instabilities of heteroepitaxial crystalline films. Lattice misfits between the substrate and the film induce elastic stresses in the film, which adjusts the shape of its free surface to reduce its total energy, sum of an elastic and a surface energy. We give a precise framework that guarantees existence of solutions to this variational problem. We show that equilibrium states can be approximated using a two-phase model for representing the surface energy. Numerical results, obtained via this approximation, are presented.
\end{abstract}

Key words. $\Gamma$-convergence, epitaxially stressed films, shape instabilities

AMS subject classifications. 49J45, 74N20

\section{Introduction}

This paper is a contribution to the mathematical formulation of morphological instabilities of interfaces induced by stress rearrangement. Stress driven rearrangement instabilities (SDRI) are observed in many branches of material sciences, such as fracture, crystal growth or corrosion. They occur for instance in the epitaxial growth of thin layers of highly strained hetero systems such as InGaAs/GaAs or SiGe/Si, in view of applications to electronic device structures. Because of these instabilities, controlling the growth of such systems is a significant challenge for the Applied Physics community (see for instance the special issue of the MSR bulletin on this topic [17]). There is also a big economic incentive as SiGe systems, for instance, would provide low-cost high-performance technology.

When a epitaxial film is grown on a (flat) substrate, if kinetic effects are neglected, the free surface of the film is flat until a critical value of the thickness is reached, after which the free surface becomes corrugated. Atomic-force microscopy images show ripples, or pits and islands of pyramidal shapes, depending on the type of alloy. 
The basic mechanism that explains this behavior is the following. The lattice misfits between the substrate and the film induce strains in the film. To release some of the elastic energy due to these strains, the atoms on the free surface of the film have the ability to move and the resulting morphology is energetically more economical.

The explanation put forward is that competition takes place between two forms of energy, the surface energy and the bulk elastic energy. The former is roughly proportional to the area of the free surface, thus favoring flat configurations. A simple asymptotic computation [13], shows that a flat free surface is unstable with respect to minimizing the bulk elastic energy of a linear elastic solid.

A model problem has been studied in [4], to understand how the stability of the uniform (flat) free surface depends on the mean thickness. In this one-dimensional formulation, the film occupies a strip $\Omega=\{0<x<1,0<y<h(x)\}$, and stable equilibria are defined as global minimizers of an energy functional that depends on the thickness $h$ and on the displacement (in the $x$ direction) in the strip $u(x)$. The problem takes the form

$$
\inf _{u \in V, h \in H} \mathcal{E}(u, h)=\inf _{u \in V, h \in H} K \int_{0}^{1} h(x)\left[u^{\prime}(x)\right]^{2} d x+\int_{0}^{1} \sqrt{1+\left[h^{\prime}(x)\right]^{2}} d x
$$

The first term in the energy expression models an elastic bulk energy induced by $u(x)$, while the second represents the length of the curve $h$. The parameter $K$ is related to the scalings of the physical constants (length of the specimen , mean thickness, elasticity constants). The larger $K$, the larger the mean thickness (all inertial effects have been neglected and the model is quasi-static).

One of the issues that stems from [4], is the choice of spaces of admissible displacements and thicknesses. In that work, which was carried out to understand computational results obtained for the above energy [9], $H$ was chosen to be the space of piecewise $\mathcal{C}^{\infty}$ positive functions, which satisfied the volume constraint

$$
\int_{0}^{1} h(x) d x=1
$$

reflecting conservation of mass during the rearrangement process. The space $V$ was simply chosen to be $x+H^{1}\left(\mathbf{S}^{1}\right)\left(\mathbf{S}^{1}\right.$ denotes the torus $\left.\mathbf{R} /(0,1)\right)$. It turns out that these spaces are not adequate neither for the analysis of the problem nor for computing purposes. In particular, if $K$ is large, the equilibrium configuration has a vertical crack that runs from the free surface to the bottom of the film. The computations did not show anything close to such a configuration, and proved (ironically) highly unstable. A sound mathematical formulation of the minimization of the energy functional should involve spaces of functions which can hence be somewhat rough; the difficulty lies then in defining the corresponding energy.

These questions are at the heart of the present paper, where we study a physically more meaningful formulation : we consider the film as a full 2-dimensional elastic solid. 
Its displacement is thus a vector-valued function $u(x, y)$. The main change concerns the modeling of the contact between the substrate and the film. In the 1-d model, strain was created by imposing boundary conditions on $u$ at the endpoints. Here, the contact affects the film in a more realistic way : a Dirichlet boundary condition is imposed at the interface between film and substrate, which models the case of a film growing on an infinitely rigid substrate. The enforcement of this boundary condition is what causes the film to be strained, i.e., what generates elastic energy.

Additionally, we make the following assumptions. Firstly, we assume that the admissible free surfaces are graphs of lower semi-continuous functions. This space is endowed with a natural topology, for which sequences of free surfaces with uniformly bounded length are compact.

Secondly, the configuration is supposed to be 1-periodic in the $x$-direction and the displacements are periodic up to a linear displacement (the displacement in the substrate).

Thirdly, we assume as in [4] that the film is made of a linear elastic material with homogeneous Hooke's law A. Finally, we assume that the substrate is infinitely large with respect to the film and occupies the region $\mathbf{S}^{1} \times(-\infty, 0]$. The contact between the substrate and the film costs surface energy, and the corresponding surface tension is denoted by $\sigma_{s}$.

This work is devoted to giving a "sound mathematical formulation" for finding the equilibrium configuration. We define an energy $E(\Omega, u)$ for graphs $\Omega$ of l.s.c. functions and for displacements $u$. We show that a minimizer exists and that it can be approximated by "smooth" thicknesses, namely by thicknesses which are Lipschitz. It was observed in [4], that when piecewise smooth thicknesses converge to a configuration with a vertical crack, the length of the crack has to be counted twice in the limiting energy. Our present formulation conveys the same feature.

We also propose an approximation scheme for computing minimizers. It is based on a diffusive two-phase model, one phase representing the film, while the other represents the void above the free surface. A Cahn-Hilliard energy [1, 5, 15] approximates the length of the free surface. The total energy $E_{\varepsilon}$ depends on the displacement $u$ and on a marker function $v$ that takes the values $v \simeq 1$ in the film and $v \simeq 0$ in the void. The scale of the Cahn-Hilliard approximation $\varepsilon$ controls the width of the transition zone between the two phases We show that, as $\varepsilon \rightarrow 0$, a minimizing graph for $E$ can be recovered as the set of points where the sequence of approximate markers $v_{\varepsilon} \rightarrow 1$. We give some numerical examples, based on the minimization of $E_{\varepsilon}$. This method is inspired by the approximation techniques introduced by [3] for a free discontinuity problem in the context of image processing, using $\Gamma$-convergence $[1,6]$. The computations of elastically stressed binary alloys of Leo et al [14] use a related approach. Muller and Grant [18] introduce a similar Ginzburg-Landau approach to study numerically, 
in two and three dimensions, the Grinfeld instability of the free interface of a nonhydrostatically stressed solid. For a concise and comprehensive review about the $\Gamma-$ convergence and the $\Gamma$-limit of the Cahn-Hilliard free energy we refer to [1].

We do not consider in this paper the anisotropic or so-called crystalline case, where the surface energy also depends on the orientation of the surface of the crystal. However, this can easily be done by introducing a convex 1-homogeneous function $\varphi(\nu)$ of the normal vector to the interface, as a weight in the lengths in section 2.1 (i.e., for instance, replacing $\mathcal{H}^{1}(\partial \Omega)$ with $\int_{\partial \Omega} \varphi(\nu(x)) d \mathcal{H}^{1}(x)$ where $\nu(x)$ is the normal vector to $\partial \Omega$ at $x$ ). In the same way, the approximation result of section 3 holds if we replace $|\nabla v(x, y)|^{2}$ with $\varphi(\nabla v(x, y))^{2}$ in (10). However, performing numerical computations is a much harder task in the crystalline case, since the physics require $\varphi$ to be singular (only Lipschitz-continuous).

The paper is organized as follows : in Section 2 the energy functional $E$ is defined in details and the lower semi-continuity of the surface energy is stated (Lemma 1). Existence of a minimizer is then proved (Theorem 1). In Section 3, we introduce the approximating energies $E_{\varepsilon}$ and we give the main theorem of this paper, Theorem 2, that states $\Gamma$-convergence of the energies $E_{\varepsilon}$ towards $E$. Numerical examples using the approximating energies $E_{\varepsilon}$ are given in Section 4 . The proofs of Lemma 1 and 2 fill in Sections 5 and 6, respectively. Finally, the Appendix groups a few results about functions with bounded variation, that are used in the text.

We do not address the issue of stability with respect to the mean thickness. These aspects will be treated subsequently.

\section{Statement of the problem and existence of a minimizer}

In the whole paper, $Q$ denotes the 2-dimensional space $\mathbf{S}^{1} \times \mathbf{R}\left(\mathbf{S}^{1}=\mathbf{R} / \mathbf{Z}\right), Q^{+}=$ $\mathbf{S}^{1} \times(0,+\infty)$, and for any $a>0, Q^{a}=\mathbf{S}^{1} \times(0, a)$. The canonical projection from $\mathbf{R}^{2}$ onto $Q$ will be denoted by $\pi$, however, in some non ambiguous situations it will not be explicitly mentioned.

We will denote by $\mathcal{G}$ the set of all open subsets $\Omega$ of $Q$ that are the sub-graph of a non-negative 1.s.c. function $h: \mathbf{S}^{1} \rightarrow[0,+\infty)$ :

$$
\Omega \in \mathcal{G} \Longleftrightarrow \exists h: \mathbf{S}^{1} \rightarrow[0,+\infty) \text { 1.s.c., } \Omega=\{(x, y) \in Q: y<h(x)\} \text {. }
$$

Note that if $\Omega \in \mathcal{G}, \partial \Omega \subset \overline{Q^{+}}=Q^{+} \cup\left(\mathbf{S}^{1} \times\{0\}\right)$. Stating that $\Omega \in \mathcal{G}$ is equivalent to saying that $Q^{-}=Q \backslash \overline{Q^{+}} \subseteq \Omega$ and that for any $(x, y) \in \Omega,\{x\} \times(-\infty, y] \subset \Omega$. We also denote by $\mathcal{G}_{L} \subset \mathcal{G}$ the sub-graphs of non-negative Lipschitz functions.

If $\left(\Omega_{n}\right)_{n \geq 1}$ is a sequence of open sets, we say that it converges to $\Omega$ as $n$ goes to infinity if it converges to $\Omega$ in the Hausdorff-complement topology, i.e. if $\Omega^{c}=Q \backslash \Omega$ is the Hausdorff limit of the complements $\Omega_{n}^{c}$. We observe that $\mathcal{G}$ is closed in the set of 
all open subsets of $Q$, indeed, if $\Omega_{n}$ are the sub-graphs of functions $h_{n}$ and $\Omega_{n}$ converge to $\Omega$ as $n$ goes to infinity, then $\Omega$ is the sub-graph of the function

$$
h(x)=\inf _{x_{n} \rightarrow x} \liminf _{n \rightarrow \infty} h_{n}\left(x_{n}\right)
$$

in fact we have $\mathcal{G}=\overline{\mathcal{G}_{L}}$.

\subsection{The surface energy}

Given $\sigma_{c}, \sigma_{s}>0$, we define the surface energy of a regular domain $\Omega \in \mathcal{G}_{L}$ as

$$
L^{0}(\Omega)=\sigma_{c} \mathcal{H}^{1}\left(\partial \Omega \cap Q^{+}\right)+\sigma_{s} \mathcal{H}^{1}\left(\partial \Omega \backslash Q^{+}\right) .
$$

(Notice that $\partial \Omega \backslash Q^{+}=\partial \Omega \cap\left(\mathbf{S}^{1} \times\{0\}\right)$.) The idea is that the part $\partial \Omega \cap Q^{+}$represents the free surface of the crystal, whose surface tension is $\sigma_{c}$, whereas $\partial \Omega \backslash Q^{+}$is the surface of the substratum that is not recovered by the crystal, and whose surface tension is $\sigma_{s}$.

We extend $L^{0}$ to $\mathcal{G}$ by setting $L^{0}(\Omega)=+\infty$ if $\Omega \in \mathcal{G} \backslash \mathcal{G}_{L}$, and define the relaxed surface energy $L: \mathcal{G} \rightarrow[0,+\infty]$ as the lower semicontinuous envelope of $L^{0}$. We have the following lemma:

Lemma 1 let $\Omega \in \mathcal{G}$, and let $h, \bar{h}$ be the l.s.c. functions such that

$$
\Omega=\{(x, y) \in Q: y<h(x)\} \text { and } \stackrel{\circ}{\Omega}=\{(x, y) \in Q: y<\bar{h}(x)\} .
$$

Then

$$
L(\Omega)=\sigma_{c}\left\{\mathcal{H}^{1}\left(\partial \bar{\Omega} \cap Q^{+}\right)+2 \sum_{x \in \mathbf{S}^{1}}(\bar{h}(x)-h(x))\right\}+\left(\sigma_{c} \wedge \sigma_{s}\right) \mathcal{H}^{1}\left(\partial \bar{\Omega} \backslash Q^{+}\right) .
$$

The proof of Lemma 1 is given in section 5. One consequence of this lemma, in particular, is the fact that if $\sigma_{s}>\sigma_{c}$, it is better to recover all of the substratum with an infinitesimal layer of crystal atoms and pay the lower surface tension $\sigma_{c}$ instead of leaving free any part of the surface of the substratum.

\subsection{The global energy functional}

We now introduce the energy

$$
E(\Omega, u)=K \int_{\Omega \cap Q^{+}} \mathbf{A} e(u)(x, y): e(u)(x, y) d x d y+L(\Omega),
$$

defined for any $\Omega \in \mathcal{G}$ and $u \in X(\Omega)$, where $X(\Omega)$ denotes the set of functions $u \in$ $L_{l o c}^{2}\left(\pi^{-1}(\Omega) ; \mathbf{R}^{2}\right), u(x, y) \equiv x$ for $y \leq 0, u(x, y)-(x, 0)$ which are 1 -periodic in $x$, and such that the linear deformation tensor (the symmetrized gradient) $e(u)$ is in $L^{2}\left(\Omega \cap Q^{+} ; \mathbf{R}^{4}\right)$. The matrix $\mathbf{A}=\left(a_{i j k l}\right)$ is a positive-definite symmetric tensor of 
order 4 (such that $a_{i j k l}=a_{j i k l}=a_{k l i j}$ for any $i, j, k, l \in\{1,2\}$ ). The scalar parameter $K$ is related to the scalings of the physical constants and it balances the influence of each term in the energy. In the rest of the paper (except in Section 4, where we illustrate the dependence on $K$ ) for simplicity we set $K=1$ without loss of generality.

\subsection{The problem}

We consider the following minimization problem

$$
\min _{\Omega \in \mathcal{G}, u \in X(\Omega)} E(\Omega, u) \quad \text { subject to: }\left|\Omega \cap Q^{+}\right|=1 .
$$

The volume constraint reflects conservation of mass: the model assumes that the relaxation of the film is much faster than the rate of deposition. We prove the following

Theorem 1 Problem (6) has a solution.

Proof. Consider $\left(\Omega_{n}, u_{n}\right)$ a minimizing sequence for (6). Since $\left|\Omega_{n} \cap Q^{+}\right|+L\left(\Omega_{n}\right)$ is bounded, $\Omega_{n} \cap Q^{+}$is uniformly bounded. Up to a subsequence (not relabeled) we may thus assume that it converges to a domain $\Omega \in \mathcal{G}$, with $\left|\Omega \cap Q^{+}\right|=1$ and, since $L$ is l.s.c.,

$$
L(\Omega) \leq \liminf _{n \rightarrow \infty} L\left(\Omega_{n}\right) .
$$

The function $e\left(u_{n}\right)$, that we extend to zero outside of $\Omega_{n}$, is uniformly bounded in $L^{2}\left(Q^{+} ; \mathbf{R}^{4}\right)$. We thus may assume it converges weakly to some function $\mathcal{E} \in$ $L^{2}\left(Q^{+} ; \mathbf{R}^{4}\right)$. In particular, we have

$$
\int_{\Omega \cap Q^{+}} \mathbf{A} \mathcal{E}(x, y): \mathcal{E}(x, y) d x d y \leq \liminf _{n \rightarrow \infty} \int_{\Omega_{n} \cap Q^{+}} \mathbf{A} e\left(u_{n}\right)(x, y): e\left(u_{n}\right)(x, y) d x d y .
$$

Remark. It can be shown that $\mathcal{E}=0$ a.e. in $Q \backslash \Omega$. Indeed, if $K$ is the Hausdorff limit of some converging subsequence of $\left(\partial \Omega_{n}\right)_{n \geq 1}$, one can prove that $\mathcal{H}^{1}(K)<+\infty$, thus $|K|=0$, and that $\Omega \cup K$ is the Hausdorff limit of $\bar{\Omega}_{n}$, or equivalently that the domains $Q \backslash \bar{\Omega}_{n}$ converge to $Q \backslash(\Omega \cup K)$ (see section 5.1 for details). Thus $\mathcal{E}=0$ in $Q \backslash(\Omega \cup K)$ and since $|K|=0$, a.e. in $Q \backslash \Omega$.

Let $A \subset \Omega$ be a Lipschitz sub-graph, with $A \cap Q^{+} \subset \subset \Omega$. (For simplicity we assume $A \supseteq Q^{-}$, although it is not essential.) For $n$ large enough, $A \subset \Omega_{n}$ and $u_{n}$ is defined on $\pi^{-1}(A)$. By Korn's inequality, since $u_{n}(x, y)-(x, 0) \equiv 0$ for $y \leq 0$ and since $\partial A$ is Lipschitz, there exist $c=c(A)$ and $c^{\prime}=c^{\prime}(A, \mathbf{A})$ such that

$$
\int_{A \cap Q^{+}}\left|u_{n}(x, y)\right|^{2} d x d y \leq c \int_{A \cap Q^{+}} e\left(u_{n}\right)(x, y): e\left(u_{n}\right)(x, y) d x d y \leq c^{\prime} E\left(\Omega_{n}, u_{n}\right) .
$$

Thus $u_{n}$ is uniformly bounded on $A \cap Q^{+}$, so that some subsequence of $\left(u_{n}\right)$ weakly converges (in fact, strongly) in $L^{2}\left(A ; \mathbf{R}^{2}\right)$ to some function $u$. Since clearly $u(x, y)-$ 
$(x, 0) \equiv 0$ for $y \leq 0$ and $e(u)=\mathcal{E}$, the limit point $u$ is unique and the whole sequence $\left(u_{n}\right)$ converges to $u$.

Since it holds for any Lipschitz sub-graph $A \cap Q^{+} \subset \subset \Omega$, this shows that there exists $u \in L_{l o c}^{2}\left(\pi^{-1}(\Omega) ; \mathbf{R}^{2}\right), 1$-periodic in $x$, with $u(x, y)-(x, 0) \equiv 0$ for $y \leq 0$, and such that $\mathcal{E}=e(u)$ in the distributional sense in $\Omega$. With (7) and (8), we conclude that $u \in X(\Omega)$ and that $(\Omega, u)$ is a solution of problem $(6)$.

\section{An approximation scheme for problem (6)}

Given a (small) scale parameter $\varepsilon>0$, we now introduce the following approximation of the energy $E$. We first choose $\eta_{\varepsilon}>0$ such that $\eta_{\varepsilon}=o(\varepsilon)$ as $\varepsilon$ goes to zero. Then we let

$$
E_{\varepsilon}(v, u)=\int_{Q^{+}}\left(v(x, y)+\eta_{\varepsilon}\right) \mathbf{A} e(u)(x, y): e(u)(x, y) d x d y+L_{\varepsilon}(v)
$$

where

$$
L_{\varepsilon}(v)=2 \sigma_{c}\left(\frac{4 \varepsilon}{\pi^{2}} \int_{Q^{+}}|\nabla v(x, y)|^{2} d x d y+\frac{1}{\varepsilon} \int_{Q^{+}} v(x, y)(1-v(x, y)) d x d y\right),
$$

for $v \in H^{1}\left(Q^{+}\right)$satisfying $0 \leq v(x, y) \leq 1, \partial_{y} v(x, y) \leq 0$ a.e. in $Q^{+}$, and $v \geq v_{s}$ on $S^{1} \times\{0\}$, and $u \in H_{l o c}^{1}\left(\mathbf{R} \times \mathbf{R}_{+} ; \mathbf{R}^{2}\right)$ such that $u(x, y)-(x, 0)$ is 1 -periodic in $x$ and vanishes on $\mathbf{R} \times\{0\}$. The constant $v_{s} \in(0,1]$ is given by

$$
\int_{0}^{v_{s}} \sqrt{t(1-t)} d t=\frac{\sigma_{c} \wedge \sigma_{s}}{\sigma_{c}} \int_{0}^{1} \sqrt{t(1-t)} d t=\frac{\sigma_{c} \wedge \sigma_{s}}{\sigma_{c}} \frac{\pi}{8} .
$$

On the other hand, if $v, u$ do not satisfy these properties we set $E_{\varepsilon}(v, u)=+\infty$.

It is well known that the Cahn-Hilliard energy $L_{\varepsilon}$ is an approximation, in the sense of $\Gamma$-convergence, of the perimeter [15]. In this particular setting we show the following result, which strictly speaking is not a result of $\Gamma$-convergence, but has the same practical consequences for the computation of minimizers of $E$.

Theorem 2 Let $\left(\varepsilon_{n}\right)_{n \geq 1}$ be an arbitrary sequence of positive numbers with $\varepsilon_{n} \downarrow 0$ as $n \rightarrow \infty$.

(i) Let $\left(v_{n}, u_{n}\right)$ be functions such that

$$
\sup _{n \geq 1} E_{\varepsilon}\left(v_{n}, u_{n}\right)<+\infty
$$

and $\sup _{n \geq 1} \int_{Q^{+}} v_{n}(\xi) d \xi<+\infty$. Then there exist $\Omega \in \mathcal{G}, u \in X(\Omega)$, and a subsequence of $\left(v_{n}, u_{n}\right)_{n \geq 1}$, still denoted by $\left(v_{n}, u_{n}\right)$, such that $v_{n} \rightarrow \chi_{\Omega}$ a.e. in $Q^{+}, u_{n} \rightarrow u$ in $L_{l o c}^{2}\left(\pi^{-1}(\Omega) ; \mathbf{R}^{2}\right)$ as $n$ goes to infinity, and

$$
E(\Omega, u) \leq \liminf _{n \rightarrow \infty} E_{\varepsilon_{n}}\left(v_{n}, u_{n}\right) .
$$


(ii) Let $\Omega \in \mathcal{G}, u \in X(\Omega)$. Then there exists a sequence $\left(v_{n}, u_{n}\right)_{n \geq 1}$ such that $v_{n} \rightarrow \chi_{\Omega}$ a.e. in $Q^{+}, u_{n} \rightarrow u$ in $L_{\text {loc }}^{2}\left(\Omega \cap Q^{+} ; \mathbf{R}^{2}\right)$ as $n$ goes to infinity, and

$$
\limsup _{n \rightarrow \infty} E_{\varepsilon_{n}}\left(v_{n}, u_{n}\right) \leq E(\Omega, u)
$$

Moreover, we can assume that for all $n, \int_{Q^{+}} v_{n}(x, y) d x d y=\left|\Omega \cap Q^{+}\right|$.

In particular, this theorem shows that if $v_{\varepsilon}, u_{\varepsilon}$ are minimizers of $E_{\varepsilon}$, subject to the constraint $\int_{Q^{+}} v_{\varepsilon}(x, y) d x d y=1$, then, to each limit point $u$ of $\left(u_{\varepsilon}\right)_{\varepsilon>0}$ corresponds a set $\Omega$ with $\left|\Omega \cap Q^{+}\right|=1$, such that $(\Omega, u)$ is a solution of problem (6).

\section{Numerical examples}

The purpose of this Section is purely illustrative : we present a few shapes obtained by minimizing approximate energies

$$
E_{\varepsilon}(v, u)=K \int_{Q^{+}}\left(v(x, y)+\eta_{\varepsilon}\right) \mathbf{A} e(u)(x, y): e(u)(x, y) d x d y+L_{\varepsilon}(v),
$$

with $L_{\varepsilon}$ given by (10). The expression (15) only differs from (9) by the presence of the parameter $K$ in front of the elastic energy. It is related to the scaling from the physical dimensions to the model problem [4], and roughly measures the mean thickness of the specimen. One expects that when $K$ is small, the term of surface energy is dominant and therefore films with a flat free-surface should minimize the energy. When $K$ becomes large, minimizers should show a corrugated free-surface.

This energy is minimized under the constraints

$$
\left\{\begin{array}{l}
u(x, 0)=(x, 0), \quad v(x, 0) \geq v_{s} \\
u-(x, 0) \text { and } v \text { are 1-periodic in } x \\
0 \leq v \leq 1, \partial_{y} v \leq 0 \text { and } \int_{Q^{1}} v=V .
\end{array}\right.
$$

For a fixed value of $\varepsilon$, we propose an iterative algorithm, based on a finite difference discretization, to minimize the energy (15). The computations are performed on a fixed rectangle $Q^{1}=\mathbf{S}^{1} \times(0,1)$, but the results are displayed on two periods. The domain $Q^{1}$ is discretized by a cartesian mesh : a $200 \times 200$ mesh in the computations presented here.

At the $n t h$ step of the algorithm, the values of $u_{n+1}$ are computed in a standard manner, as approximations of the solutions to the Euler equation

$$
\operatorname{div}\left(\left(v_{n}+\eta_{\varepsilon}\right) A e\left(u_{n+1}\right)\right)=0
$$

with the boundary conditions and the periodicity given above. 
For the density, our approach is inspired by algorithms for motion by mean curvature described in $[19,20]$, where in $L_{\varepsilon}$ a non-regular potential of the same form is used. Minimizers of $E_{\varepsilon}\left(., u_{n+1}\right)$ are computed as if they were stationary states of the parabolic problem

$$
\left\{\begin{array}{c}
\partial_{t} v=2 \sigma_{c}\left(\frac{8 \varepsilon}{\pi^{2}} \Delta v-1 / \varepsilon(1-2 v)\right)-A e\left(u_{n+1}\right): e\left(u_{n+1}\right)-\lambda \\
0 \leq v \leq 1, \partial_{y} v \leq 0
\end{array}\right.
$$

with the initial condition $v_{n+1}^{(0)}=v_{n}$, and where $\lambda$ is a Lagrange multiplier for the volume constraint $\int_{Q^{1}} v=V$. More precisely, we compute functions $v_{n+1}^{(k+1)} 1$-periodic in $x$ and such that $v_{n+1}^{(k+1)}(x, 0) \geq v_{s}$, approximate solutions to the following variational problem

$$
\left\{\begin{array}{l}
1 / \delta \int_{Q^{1}}\left(v_{n+1}^{(k+1)}-v_{n+1}^{(k)}\right) \varphi \\
+2 \sigma_{c}\left(\frac{8 \varepsilon}{\pi^{2}} \int_{Q^{1}} \nabla v_{n+1}^{(k+1)} \nabla \varphi+1 / \varepsilon \int_{Q^{1}}\left(1-v_{n+1}^{(k+1)}\right) \varphi\right) \\
+\int_{Q^{1}}\left(\lambda+A e\left(u_{n+1}\right): e\left(u_{n+1}\right)\right) \varphi=0
\end{array}\right.
$$

where the test functions $\varphi$ are 1 -periodic in $x$ and satisfy $\varphi(x, 0)=0$. The constraints $0 \leq v_{n+1}^{(k+1)} \leq 1$ and $\partial_{y} v_{n+1}^{(k+1)} \leq 0$ are then enforced at each iteration by a simple truncation. The parameter $\delta$ corresponds to an artificial time step in view of (17), and is required to be smaller than $\varepsilon / 2 \sigma_{c}$. In this manner, the variational problem above is the Euler-Lagrange equation of a convex minimization problem, and therefore existence of a solution $v_{n+1}^{(k+1)}$ is guaranteed.

One of the virtues of this method is that the energy $E_{\varepsilon}$ is decreasing in the course of the algorithm. Indeed, since (18) expresses that $v_{n+1}^{(k+1)}$ is the minimizer of the energy $E_{\varepsilon}\left(\cdot, u_{n+1}\right)+1 / 2 \delta \int_{Q^{1}}\left|\cdot-v_{n+1}^{(k)}\right|^{2}$, one easily checks that

$$
E_{\varepsilon}\left(v_{n+1}^{(k+1)}, u_{n+1}\right)-E_{\varepsilon}\left(v_{n+1}^{(k)}, u_{n+1}\right) \leq-1 / 2 \delta \int_{Q^{1}}\left|v_{n+1}^{(k+1)}-v_{n+1}^{(k)}\right|^{2}
$$

When $E_{\varepsilon}\left(v_{n+1}^{(k)}, u_{n+1}\right)$ has become stationary, we assign $v_{n+1}=v_{n+1}^{(k)}$.

This method for computing equilibrium shapes thus depends on several parameters: $\varepsilon, \eta_{\varepsilon}, \delta$, and the mesh-size $h$. The transition zone should be described by at least a few mesh-points. In fact, during the computations, we let $\varepsilon$ vary from 20 mesh sizes to 3 . The parameter $\eta_{\varepsilon}$ has been chosen equal to 0.001 . The Hooke's law of the film is that of an isotropic elastic material, with Lamé constants $\lambda=1$ and $\mu=2$.

We first illustrate the results obtained by this algorithm when considering a onedimensional approximation for the elastic displacement, like in (1). The approximate 
energy functionals reduce then to

$$
E_{\varepsilon}(v, u)=K \int_{Q^{1}}\left(v(x, y)+\eta_{\varepsilon}\right)\left|\partial_{x} u_{1}\right|^{2}+L_{\varepsilon}(v)
$$

where $u_{1}(x)$ denotes the average in $y$ of the $x$-component of the displacement. In this context, it was shown in [4] that for $K$ large, the optimal configuration of the free surface consisted of half circles separated by cracks. Figure 1 shows that the algorithm does capture the optimal configuration.

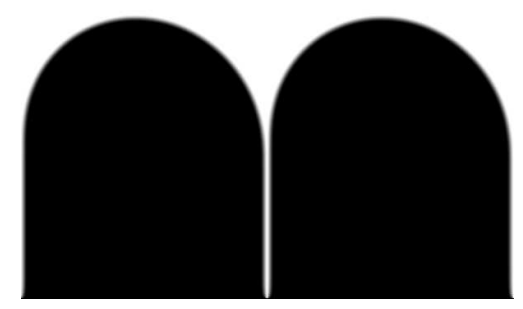

Figure 1: Equilibrium shape for the 1-d model.

The rest of the figures are pertinent to the energy (15). The value of the density on the bottom of the film has been chosen to be $v \geq v_{s}=0.99$ unless stated otherwise, and the volume of the film is constrained to be equal to $V=0.2$. In those computations the initial shape has the form $v_{0}(y)+a \sin (2 \pi n x) f(y)$. The function $v_{0}$ is the optimal profile associated with minimizing, under the volume constraint, the energy $L_{\varepsilon}$ only, see (44). If the prescribed volume of the film is $V, v_{0}$ has the expression :

$$
v_{0}(y)= \begin{cases}1 & \text { if } y \leq V-\varepsilon \\ \frac{1}{2}\left(1-\sin \frac{\pi(y-V)}{2 \varepsilon}\right) & \text { if } V-\varepsilon \leq y \leq V+\varepsilon \\ 0 & \text { if } y \geq V+\varepsilon\end{cases}
$$

The remaining term in the expression of the initial shape is a perturbation, consisting of a sinusoidal function in $x$, times a function $f$ of $y$ whose main attribute is to be concentrated in the transition zone of $v_{0}$. The choice of the $x$-dependence of the perturbation stems from a linearized stability analysis around the shape $v_{0}$, which can be shown to be a global minimizer for the energy $E_{\varepsilon}$, when $K$ is small, corresponding to the flat free-surface $\Omega=\mathbf{S}^{1} \times(0, V)$ solution to (6). These aspects will be discussed elsewhere, however.

Figure 2 shows the initial and final shapes for a computation with $K=0.5$. The initial shape is $v_{0}(y)$ plus a perturbation of amplitude $a=0.4$ and frequency $n=2$. 
The resulting free surface is flat, which is consistent with the small value of $K$. Figure 3 shows the variations of the total energy, of its elastic and surface energy components during this computation

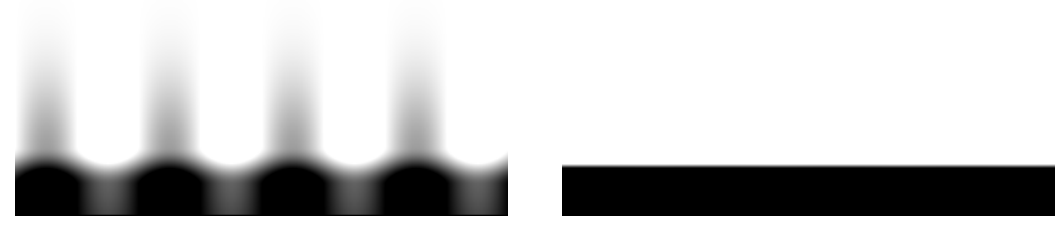

Figure 2: Initial and final shapes, $K=0.05$

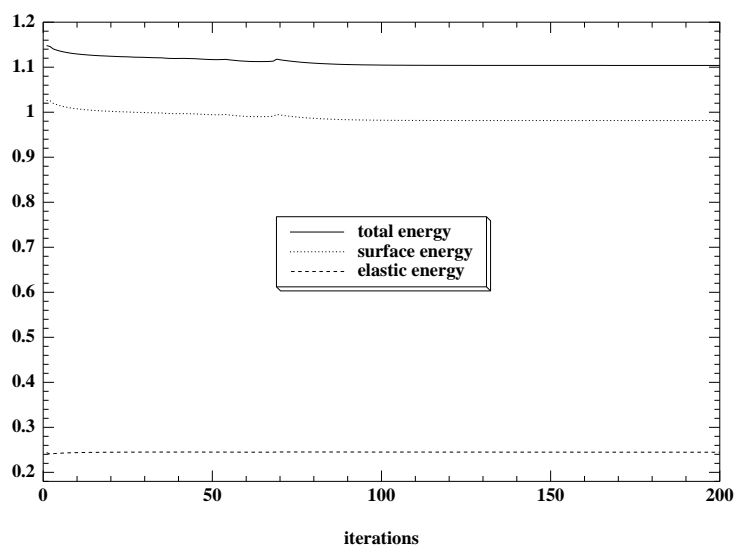

Figure 3: Energies, $K=0.5$, initial shape $v_{0}(y)+0.05 \sin (2 \pi x) f(y)$

Figure 4 shows the initial shape (with amplitude $a=0.7$ and frequency $n=1$ ), and the final shapes resulting from the algorithm when $K=6$ and for three different values of $v_{s}(0.99,0.6$ and 0.4$)$. Contrarily to the one-dimensional model, no sharp cracks are observed, rather wide zones with a nearly barren substrate are formed, while the film forms bumps in the shape of milestones. The width of the base of these milestones depends on the value of $v_{s}$ : this reflects the fact that $v_{s}$ is itself a function of the ratio $\sigma_{c} / \sigma_{s}$, and determines the cost of leaving the substrate barren. Figure 5 shows the history of energies, when $v_{s}=0.99$. Finally, we present some results that demonstrate the unstable behavior of the system for large values of $K$. Figure 6 shows various images of the configurations obtained during a computation with $K=20$, initiated with the profile $v_{0}(y)$ which is a local minimum. After a large number of iterations when nothing seems to happen to the 'flat surface', instabilities are triggered by round-off errors. One observes corrugations that finally merge together to form a unique bigger milestone. Figure 7 shows the corresponding energies. 

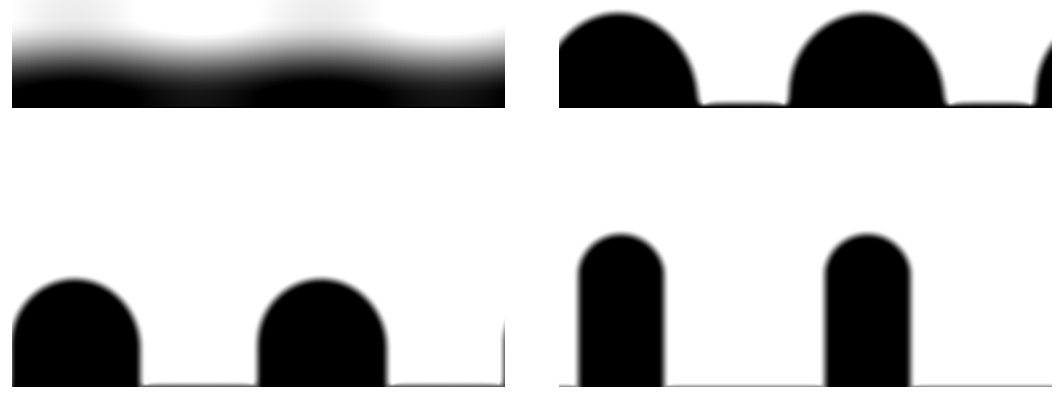

Figure 4: Initial and final shapes, $K=6$

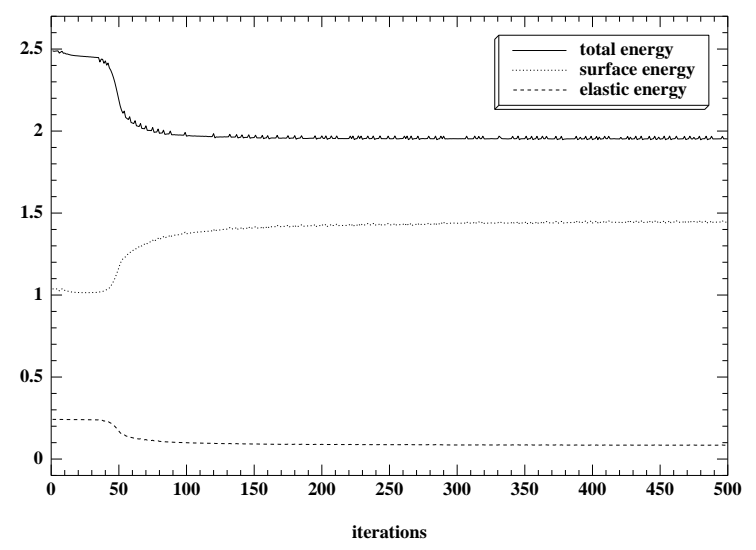

Figure 5: Energies, $K=6, v_{s}=0.99$

\section{Proof of Lemma 1}

In order to prove Lemma 1, we need to show that

(i) given any $\Omega_{n}$ converging to $\Omega$ in $\mathcal{G}$,

$$
L(\Omega) \leq \liminf _{n \rightarrow \infty} L^{0}\left(\Omega_{n}\right),
$$

and that

(ii) for any $\Omega \in \mathcal{G}$, there exists a sequence $\Omega_{n}$ converging to $\Omega$ such that

$$
\limsup _{n \rightarrow \infty} L^{0}\left(\Omega_{n}\right) \leq L(\Omega)
$$

where $L$ is defined by equation (4). 


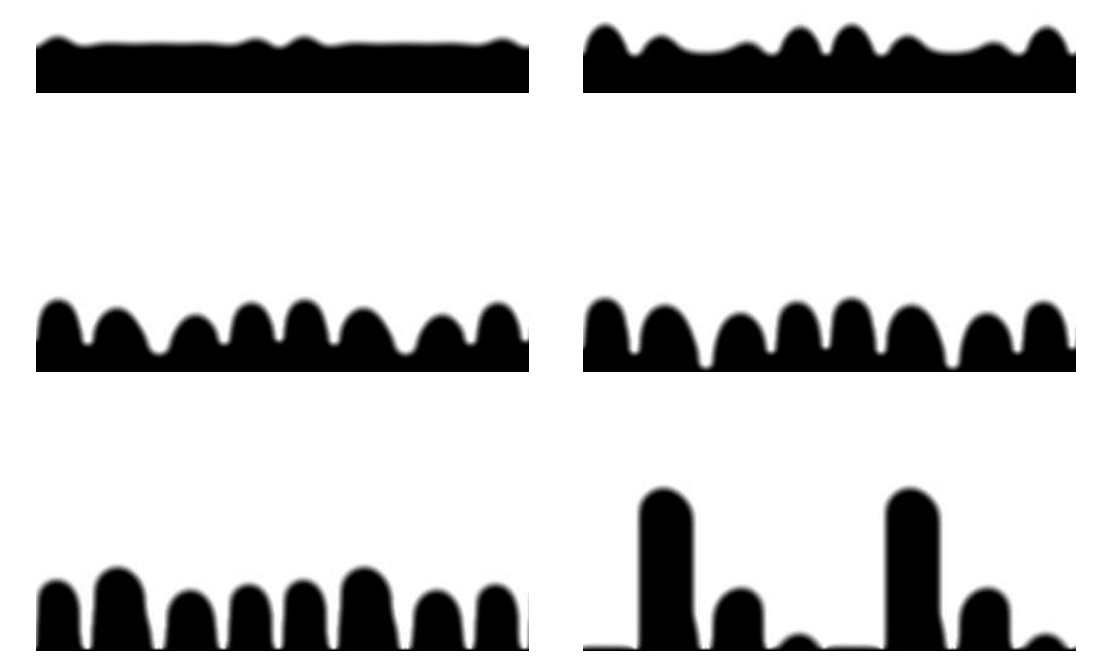

Figure 6: Shapes at iterations 1936, 1940, 1944, 1948, 2000, 2100, K=20, initial shape $v_{0}(y)$

\subsection{Proof of (i)}

To show (i), we consider $\Omega_{n}$ converging to $\Omega$ in $\mathcal{G}$. Without loss of generality we can assume that $a=\sup _{n} L^{0}\left(\Omega_{n}\right)+\left|\Omega_{n} \cap Q^{+}\right|<+\infty$ (since if $\liminf _{n \rightarrow \infty} L^{0}\left(\Omega_{n}\right)=+\infty$ there is nothing to prove, and if $\left|\Omega_{n} \cap Q^{+}\right| \rightarrow \infty$ and $L^{0}\left(\Omega_{n}\right)$ is bounded $\Omega_{n}$ converges to $Q \notin \mathcal{G})$. In particular, the $\Omega_{n}$ are sub-graphs of Lipschitz functions $h_{n}: \mathbf{S}^{1} \rightarrow[0, a)$.

Clearly, $\partial \Omega_{n} \subset \overline{Q^{a}}$, so that we may assume (by extracting a subsequence, still denoted by $\left.\left(\Omega_{n}\right)\right)$ that the boundaries $\partial \Omega_{n}$ converge in the Hausdorff metric to some compact set $K$. Notice that we easily deduce that $\bar{\Omega}_{n}$ converge to $\Omega \cup K$ in the Hausdorff sense: indeed if $\xi \in \Omega \cup K$, either $\xi \in \Omega$ and therefore $\xi \in \Omega_{n}$ for large $n$, or $\xi \in K$ and there exists $\xi_{n} \in \partial \Omega_{n} \subset \bar{\Omega}_{n}$ such that $\xi=\lim _{n \rightarrow \infty} \xi_{n}$. Conversely if $\xi_{n} \in \bar{\Omega}_{n}$ for all $n$ and converge to some $\xi$ as $n$ goes to infinity, if $\xi \notin \Omega$ then there exists $\xi_{n}^{\prime} \in Q \backslash \Omega_{n}$ with $\xi=\lim _{n \rightarrow \infty} \xi_{n}^{\prime}$ (since $Q \backslash \Omega_{n}$ converge to $Q \backslash \Omega$ in the Hausdorff sense) and there exists $\xi_{n}^{\prime \prime} \in\left[\xi_{n}, \xi_{n}^{\prime}\right) \cap \partial \Omega_{n}$ : since $\xi_{n}^{\prime \prime} \rightarrow \xi$, we deduce that $\xi \in K$. In a similar way we can show that $\partial \Omega \subset K$.

Then, invoking Golab's theorem (see $[12,16,8]$ ), $\partial \Omega_{n}$ being a sequence of uniformly bounded one-dimensional compact connected sets, we get that

$$
\mathcal{H}^{1}(\partial \Omega) \leq \mathcal{H}^{1}(K) \leq \liminf _{n \rightarrow \infty} \mathcal{H}^{1}\left(\partial \Omega_{n}\right)<+\infty .
$$

Moreover, the proofs of Golab's theorem based on measure density arguments (see [16]) also show that the measures $\mu_{n}=\mathcal{H}^{1}\left\llcorner\partial \Omega_{n}\right.$, up to a subsequence (not 


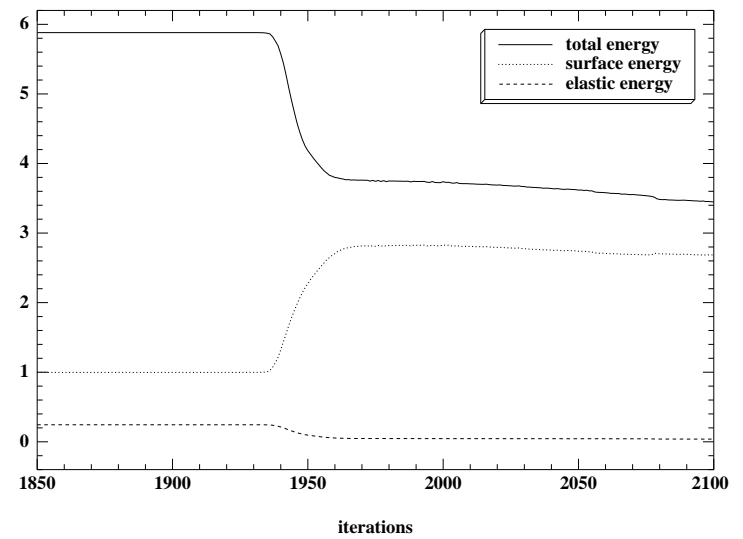

Figure 7: Energies, $K=20$, initial shape $y(x)=1$

relabeled), converge weakly-* to a measure $\mu$ supported by $K$ such that

$$
\mathcal{H}^{1}\llcorner K \leq \mu \text {. }
$$

We define the l.s.c. functions $h, \bar{h}: \mathbf{S} \rightarrow[0, a]$ as $h(x)=\min \{y:(x, y) \notin \Omega\}$ (resp., $\bar{h}(x)=\min \{y:(x, y) \notin \stackrel{\circ}{\Omega}\})$ so that (3) holds. Since $\Omega \subseteq \stackrel{\circ}{\Omega}, h \leq \bar{h}$.

We first observe that $\partial \Omega$ is the disjoint union of $\partial \bar{\Omega}$ and $\left(\bigcup_{x \in \mathbf{S}^{1}}\{x\} \times[h(x), \bar{h}(x))\right.$. Together with (21), this implies that $h(x)=\bar{h}(x)$ except for at most countably many $x \in \mathbf{S}^{1}$. As a matter of fact, $\partial \bar{\Omega}=\bar{\Omega} \backslash \stackrel{\circ}{\bar{\Omega}} \subseteq \bar{\Omega} \backslash \Omega=\partial \Omega$. Then, $\partial \Omega \backslash \partial \bar{\Omega}=\stackrel{\circ}{\Omega} \backslash \Omega$, so that by definition of $h$ and $\bar{h}$,

$$
\partial \Omega \backslash \partial \bar{\Omega}=\{(x, y) \in Q: h(x) \leq y<\bar{h}(x)\}=\bigcup_{x \in \mathbf{S}^{1}}\{x\} \times[h(x), \bar{h}(x)),
$$

which proves the claim.

We now define the measures $\nu_{n}=\sigma_{c} \mathcal{H}^{1}\left\llcorner\partial \Omega_{n} \cap Q^{+}+\sigma_{s} \mathcal{H}^{1}\left\llcorner\partial \Omega_{n} \backslash Q^{+}\right.\right.$. It is not restrictive to assume that $\nu_{n}$ converges weakly-* to a positive measure $\nu$ as $n$ goes to infinity, and we clearly have

$$
\left(\sigma_{s} \wedge \sigma_{c}\right) \mu \leq \nu \leq\left(\sigma_{s} \vee \sigma_{c}\right) \mu .
$$

Moreover, since $Q^{+}$is open and $\nu_{n}\left\llcorner Q^{+}=\sigma_{c} \mu_{n}\left\llcorner Q^{+}\right.\right.$for all $n$, we also have

$$
\nu\left\llcorner Q^{+}=\sigma_{c} \mu\left\llcorner Q^{+}\right.\right.
$$

To deduce (19), it remains to show that the one-dimensional density of $\nu$ on the set $\partial \Omega \backslash \partial \bar{\Omega}=\bigcup_{x \in \mathbf{S}^{1}}\{x\} \times[h(x), \bar{h}(x))$ is at least $2 \sigma_{c}$. Indeed, since

$$
\begin{aligned}
\liminf _{n \rightarrow \infty} L^{0}\left(\Omega_{n}\right) & =\liminf _{n \rightarrow \infty} \nu_{n}(Q) \geq \nu(Q)=\nu(K) \\
& \geq \nu(\partial \Omega)=\nu\left(\partial \bar{\Omega} \cap Q^{+}\right)+\nu\left(\partial \bar{\Omega} \backslash Q^{+}\right)+\nu(\partial \Omega \backslash \partial \bar{\Omega}),
\end{aligned}
$$


it will imply together with (22), (23) and (24) that

$$
\begin{aligned}
& \liminf _{n \rightarrow \infty} L^{0}\left(\Omega_{n}\right) \geq \\
& \sigma_{c} \mathcal{H}^{1}\left(\partial \bar{\Omega} \cap Q^{+}\right)+\left(\sigma_{c} \wedge \sigma_{s}\right) \mathcal{H}^{1}\left(\partial \bar{\Omega} \backslash Q^{+}\right)+2 \sigma_{c} \mathcal{H}^{1}(\partial \Omega \backslash \partial \bar{\Omega})=L(\Omega) .
\end{aligned}
$$

It remains therefore to show that for $\mathcal{H}^{1}$-almost any $\xi=(x, y) \in \partial \Omega \backslash \partial \bar{\Omega}$,

$$
\limsup _{\rho \downarrow 0} \frac{\nu(\overline{B(\xi, \rho)})}{2 \rho} \geq 2 \sigma_{c} .
$$

Choose such a $\xi$, with $h(x)<y$ (we exclude the case $h(x)=y$ since $\{(x, h(x)): h(x)<$ $\bar{h}(x)\}$ is at most countable, and thus $\mathcal{H}^{1}$-negligible) and $\rho>0$ small enough, so that $h(x)<y-\rho, y+\rho<\bar{h}(x)$, and $B(\xi, 2 \rho) \subset \bar{\Omega}$.

For some $\delta>0$ small enough, $(x, y-\rho-\delta) \notin \Omega$, so that there exists $\left(x_{n}, y_{n}\right) \notin \Omega_{n}$ such that $\left(x_{n}, y_{n}\right) \rightarrow(x, y-\rho-\delta)$ as $n$ goes to infinity. If $n$ is large enough, $y_{n}<y-\rho$, and $h_{n}\left(x_{n}\right)<y-\rho$, where $h_{n}$ is the Lipschitz function whose sub-graph is $\Omega_{n}$.

Fix $\varepsilon>0$ small $(\varepsilon<<\rho)$ and choose $x^{\prime}, x^{\prime \prime}$ with $x-\varepsilon<x^{\prime}<x<x^{\prime \prime}<x+\varepsilon$. Choose also $y^{\prime}, y^{\prime \prime}$ such that $\left(x^{\prime}, y^{\prime}\right),\left(x^{\prime \prime}, y^{\prime \prime}\right) \in B(\xi, 2 \rho) \backslash \overline{B(\xi, \rho)}$ and $y^{\prime}>y+\rho, y^{\prime \prime}>y+\rho$. Since the $\bar{\Omega}_{n}$ converge in the Hausdorff sense to $\Omega \cup K \supset \bar{\Omega}$, and since $\left(x^{\prime}, y^{\prime}\right),\left(x^{\prime \prime}, y^{\prime \prime}\right) \in \bar{\Omega}$, there are sequences $\left(x_{n}^{\prime}, y_{n}^{\prime}\right)$ and $\left(x_{n}^{\prime \prime}, y_{n}^{\prime \prime}\right)$ converging respectively to $\left(x^{\prime}, y^{\prime}\right)$ and $\left(x^{\prime \prime}, y^{\prime \prime}\right)$ such that $\left(x_{n}^{\prime}, y_{n}^{\prime}\right),\left(x_{n}^{\prime \prime}, y_{n}^{\prime \prime}\right) \in \bar{\Omega}_{n}$ for all $n$.

If $n$ is large enough, $x-\varepsilon<x_{n}^{\prime}<x_{n}<x_{n}^{\prime \prime}<x+\varepsilon$, and $h_{n}\left(x_{n}^{\prime}\right), h_{n}\left(x_{n}^{\prime \prime}\right)>y+\rho$, $h_{n}\left(x_{n}\right)<y-\rho$, so that the length of the graph $\partial \Omega_{n}$ of $h_{n}$ inside the ball $B(\xi, \rho)$ must be at least $2 \times 2 \rho-O\left(\varepsilon^{2}\right)$. We deduce that for large $n, \nu_{n}(\overline{B(\xi, \rho)}) \geq 2 \sigma_{c} 2 \rho-O\left(\varepsilon^{2}\right)$, and therefore $\nu(\overline{B(\xi, \rho)}) \geq \lim \sup _{n \rightarrow \infty} \nu_{n}(\overline{B(\xi, \rho)}) \geq 2 \sigma_{c} 2 \rho-O\left(\varepsilon^{2}\right)$. Sending $\varepsilon$ to 0 , we get that for any (small) $\rho>0$,

$$
\frac{\nu(\overline{B(\xi, \rho)})}{2 \rho} \geq 2 \sigma_{c}
$$

so that (25) clearly holds.

\subsection{Proof of (ii)}

We now must build, given $\Omega \in \mathcal{G}$, a sequence $\Omega_{n}$ converging to $\Omega$ satisfying (20). It is of course not restrictive to assume that $L(\Omega)<+\infty$.

We consider the l.s.c. functions $h$ and $\bar{h}$ as in (3). Since $L(\Omega)<+\infty, h$ and $\bar{h}$ are bounded and $h(x)=\bar{h}(x)$ except for an at most countable number of points $x \in \mathbf{S}^{1}$. Moreover, $h$ and $\bar{h}$ are functions of bounded variation, in the classical sense (see Appendix A, and in particular section A.2.2 and inequality (53)). They thus have a right and left limit at each point. Now, since $h=\bar{h}$ a.e., we deduce that for any $x \in \mathbf{S}^{1}, \bar{h}(x-0)=h(x-0)$ and $\bar{h}(x+0)=h(x+0)\left(\right.$ where $\left.h(x \pm 0)=\lim _{\varepsilon \downarrow 0^{+}} h(x \pm \varepsilon)\right)$. 
Now, $h$ being considered as a 1 -periodic function defined on the whole real line (meaning that we still denote by $h$ what should theoretically be $h \circ \pi$ ), for all $n \geq 1$ let $h_{n}$ be the $n$-Lipschitz 1-periodic and non-negative function defined by

$$
h_{n}(x)=\inf _{x^{\prime} \in \mathbf{R}} h\left(x^{\prime}\right)+n\left|x-x^{\prime}\right|
$$

and define $\Omega_{n} \in \mathcal{G}$ as the sub-graph of $h_{n}$. Notice that since $h$ is l.s.c., the infimum in (26) is reached. Since $h_{n} \leq h, \Omega_{n} \subseteq \Omega$. It is also well-known that for all $x$, $h_{n}(x) \uparrow h(x)$ as $n \rightarrow \infty$. Let us show that $\Omega$ is the limit of the sequence $\left(\Omega_{n}\right)_{n \geq 1}$. Let $A$ be the limit of some converging subsequence $\left(\Omega_{n_{k}}\right)_{k \geq 1}$. Since $\Omega_{n}$ is increasing it is not difficult to show that $A$ is the limit of the whole sequence $\left(\Omega_{n}\right)_{n \geq 1}$. Clearly, $A \subseteq \Omega$, and we want to show the reverse inequality. Let $\xi=(x, y) \notin A$. There exist $\xi_{n}=\left(x_{n}, y_{n}\right) \notin \Omega_{n}$, such that $\xi_{n} \rightarrow \xi$ as $n \rightarrow \infty$. Since the infimum is reached in (26), there exist $x_{n}^{\prime}$ such that

$$
y_{n} \geq h_{n}\left(x_{n}\right)=h\left(x_{n}^{\prime}\right)+n\left|x_{n}-x_{n}^{\prime}\right|
$$

for every $n$. In particular since $\left(y_{n}\right)_{n \geq 1}$ is bounded, $x_{n}^{\prime} \rightarrow x$ as $n$ goes to infinity. Since $h$ is l.s.c., we deduce that

$$
h(x) \leq \liminf _{n \rightarrow \infty} h\left(x_{n}^{\prime}\right) \leq \liminf _{n \rightarrow \infty} y_{n}=y,
$$

so that $\xi \notin \Omega$ : therefore $\Omega \subseteq A$, and we have proved that $\Omega$ is the limit of $\Omega_{n}$.

Now, we show that (20) holds for the sequence $\Omega_{n}$. We have $\partial \Omega_{n}=\partial \bar{\Omega}_{n}=$ $\left\{\left(x, h_{n}(x)\right): x \in \mathbf{S}^{1}\right\}$.

We split $\partial \Omega_{n}$ into two parts, $\partial \Omega_{n} \cap \partial \Omega$ and $\partial \Omega_{n} \backslash \partial \Omega$. First notice that $\partial \Omega \cap \partial \Omega_{n}$ is essentially equal to $\partial \bar{\Omega} \cap \partial \Omega_{n}$. Indeed, let $(x, y) \in \partial \Omega_{n} \cap(\partial \Omega \backslash \partial \bar{\Omega})$. We have $y=h_{n}(x) \leq h(x)$, and $h(x) \leq y<\bar{h}(x)$, thus $y=h(x)$ and $h(x)<\bar{h}(x)$, but we know that this happens for at most a countable number of points $x$. Thus, $\partial \Omega_{n} \cap(\partial \Omega \backslash \partial \bar{\Omega})$ is at most countable.

Now, suppose that $h_{n}(x)=0$ : then there exists $x^{\prime} \in \mathbf{R}$ such that $h\left(x^{\prime}\right)+n\left|x-x^{\prime}\right|=$ 0 . Since $h$ is non-negative, it implies that $x^{\prime}=x$ and $h(x)=0$. On the other hand, if $h(x)=0$, since $0 \leq h_{n} \leq h, h_{n}(x)=0$. We deduce that $\partial \Omega_{n} \backslash Q^{+}=\partial \Omega \backslash Q^{+}$for any $n \geq 1$, moreover this set is essentially equal to $\partial \bar{\Omega} \backslash Q^{+}$.

We thus deduce that, for every $n$,

$$
\begin{aligned}
L^{0}\left(\Omega_{n}\right) & =\sigma_{s} \mathcal{H}^{1}\left(\partial \Omega_{n} \backslash Q^{+}\right)+\sigma_{c} \mathcal{H}^{1}\left(\partial \Omega_{n} \cap Q^{+}\right) \\
& =\sigma_{s} \mathcal{H}^{1}\left(\partial \bar{\Omega} \backslash Q^{+}\right)+\sigma_{c} \mathcal{H}^{1}\left(\partial \bar{\Omega} \cap \partial \Omega_{n} \cap Q^{+}\right)+\sigma_{c} \mathcal{H}^{1}\left(\partial \Omega_{n} \backslash \partial \Omega\right) .
\end{aligned}
$$

We need now to estimate $\mathcal{H}^{1}\left(\partial \Omega_{n} \backslash \partial \Omega\right)$. Notice that clearly, $\partial \Omega_{n} \backslash \partial \Omega=\left\{\left(x, h_{n}(x)\right)\right.$ : $\left.h_{n}(x)<h(x)\right\}$. Since $\partial \Omega_{n} \backslash \partial \Omega$ is open, it can be written as a disjoint union of open connected arcs:

$$
\partial \Omega_{n} \backslash \partial \Omega=\bigcup_{k \in K}\left\{\left(x, h_{n}(x)\right): x \in I_{k}\right\}
$$


where the set $K$ is finite or countable, and for each $k, I_{k}=\left(a_{k}, b_{k}\right) \subset \mathbf{S}^{1}$ is an open interval.

Fix $k \in K$ and consider such a $I_{k}$. We claim that for all $x \in I_{k}$,

$$
h_{n}(x)=\min \left\{h\left(a_{k}\right)+n\left|x-a_{k}\right|, h\left(b_{k}\right)+n\left|x-b_{k}\right|\right\} .
$$

In order to simplify the notations we temporarily drop the subscript $k$, and let therefore $I=(a, b)=I_{k}$. We can consider $(a, b)$ as an interval in $\mathbf{R}$, with $a<b$. Since $h_{n}$ is $n$-Lipschitz, $h_{n}(x)-h_{n}(a) \leq n|x-a|$ so that for any $x, h_{n}(x) \leq h_{n}(a)+n|x-a| \leq$ $h(a)+n|x-a|$. Thus, $h_{n}(x) \leq \min \{h(a)+n|x-a|, h(b)+n|x-b|\}$.

Assume now that there exists $x \in I$ such that the inequality is strict, and let $x^{\prime} \in \mathbf{R}$ be a point where the infimum is reached in (26). We have $h\left(x^{\prime}\right)+n\left|x-x^{\prime}\right|=h_{n}(x)<$ $h(a)+n|x-a|$, so that if $x^{\prime} \leq a, h\left(x^{\prime}\right)+n\left|a-x^{\prime}\right|<h(a)$, but this is in contradiction with the fact that $h_{n}(a)=h(a)$ : thus $x^{\prime}>a$. In the same way, we show that $x^{\prime}<b$, so that $x^{\prime} \in I$.

But if $x^{\prime \prime}$ is such that $h_{n}\left(x^{\prime}\right)=h\left(x^{\prime \prime}\right)+n\left|x^{\prime}-x^{\prime \prime}\right|$, then,

$$
\begin{aligned}
h_{n}(x) & \leq h_{n}\left(x^{\prime \prime}\right)+n\left|x^{\prime \prime}-x\right| \quad \text { (since } h_{n} \text { is } n \text {-Lipschitz) } \\
& \leq h\left(x^{\prime \prime}\right)+n\left|x^{\prime \prime}-x^{\prime}\right|+n\left|x^{\prime}-x\right| \\
& =h_{n}\left(x^{\prime}\right)+n\left|x^{\prime}-x\right| \\
& \leq h\left(x^{\prime}\right)+n\left|x^{\prime}-x\right|=h_{n}(x),
\end{aligned}
$$

so that $h_{n}\left(x^{\prime}\right)=h\left(x^{\prime}\right)$ and $x^{\prime} \notin I$. Therefore $x$ can not exist, and (28) holds for every point in $I=I_{k}$.

We deduce an estimate for the contribution of the interval $I=I_{k}$ to the length of $\partial \Omega_{n} \backslash \partial \Omega$. We consider the two cases

(a) for all $x \in I, h_{n}(x)=h(a)+n(x-a)$ (or $\left.h_{n}(x)=h(b)+n(b-x)\right)$;

(b) there exists $c \in I$ such that $h_{n}(x)=h(a)+n(x-a)$ for all $x \in(a, c]$ and $h_{n}(x)=h(b)+n(b-x)$ for all $x \in[c, b)$.

In the first case, the graph of $h_{n}$ in $\bar{I} \times \mathbf{R}$ is a straight line going from $(a, h(a))$ to $(b, h(b))$, while the boundary $\partial \Omega \cap(\bar{I} \times \mathbf{R})$ contains a curve connecting these two points. This curve is made of a possible piece of straight line going from $(a, h(a))$ to $(a, h(a+0))$, then a curve going from $(a, h(a+0))$ to $(b, h(b-0))$, essentially contained in $\partial \bar{\Omega} \cap(I \times \mathbf{R})$, and then another possible piece of straight line going from $(b, h(b-0))$ to $(b, h(b))$, so that

$$
\mathcal{H}^{1}\left(\partial \Omega_{n} \cap\left(\bar{I}_{k} \times \mathbf{R}\right)\right) \leq h\left(a_{k}+0\right)-h\left(a_{k}\right)+h\left(b_{k}-0\right)-h\left(b_{k}\right)+\mathcal{H}^{1}\left(\partial \bar{\Omega} \cap\left(I_{k} \times \mathbf{R}\right)\right)
$$

In the case (b), the graph of $h_{n}$ in $\bar{I} \times \mathbf{R}$ is made of two straight lines, one going from $(a, h(a))$ to $\left(c, h_{n}(c)\right)$ and the other from $\left(c, h_{n}(c)\right)$ to $(b, h(b))$. The boundary 
$\partial \Omega \cap(\bar{I} \times \mathbf{R})$ contains a curve connecting $(a, h(a))$ to $(b, h(b))$, and passing through the point $(c, \bar{h}(c))$, and since $\max \{h(a), h(b)\}<h_{n}(c)<\bar{h}(c),(29)$ still holds.

Summing over $k \in K$, we deduce that

$$
\mathcal{H}^{1}\left(\partial \Omega_{n} \backslash \partial \Omega\right) \leq \mathcal{H}^{1}\left(\partial \bar{\Omega} \cap \bigcup_{k \in K}\left(I_{k} \times \mathbf{R}\right)\right)+\sum_{k \in K} h\left(a_{k}+0\right)-h\left(a_{k}\right)+h\left(b_{k}-0\right)-h\left(b_{k}\right) .
$$

Now, it is possible to show that for any $x$, if for instance $h(x+0) \geq h(x-0)$, then (recalling that $h$ and $\bar{h}$ are l.s.c.) $\bar{h}(x)=h(x-0)$ and

$$
\begin{aligned}
h(x+0)+h(x-0)-2 h(x) & =\bar{h}(x+0)-\bar{h}(x)+2 \bar{h}(x)-2 h(x) \\
& =\mathcal{H}^{1}(\partial \bar{\Omega} \cap(\{x\} \times \mathbf{R}))+2(\bar{h}(x)-h(x)) .
\end{aligned}
$$

From (30) and (31), we deduce that

$$
\mathcal{H}^{1}\left(\partial \Omega_{n} \backslash \partial \Omega\right) \leq \mathcal{H}^{1}\left(\partial \bar{\Omega} \cap \bigcup_{k \in K}\left(\bar{I}_{k} \times \mathbf{R}\right)\right)+2 \mathcal{H}^{1}(\partial \Omega \backslash \partial \bar{\Omega}) .
$$

Now, it is clear that $\partial \bar{\Omega} \cap \bigcup_{k \in K}\left(\bar{I}_{k} \times \mathbf{R}\right)$ is contained in $Q^{+}$, and that, up to an at most countable number of points, it is disjoint from $\partial \bar{\Omega} \cap \partial \Omega_{n} \cap Q^{+}$. So that we can deduce from (27) and (32) that

$$
L^{0}\left(\Omega_{n}\right) \leq \sigma_{s} \mathcal{H}^{1}\left(\partial \bar{\Omega} \backslash Q^{+}\right)+\sigma_{c}\left(\mathcal{H}^{1}\left(\partial \bar{\Omega} \cap Q^{+}\right)+2 \mathcal{H}^{1}(\partial \Omega \backslash \partial \bar{\Omega})\right)=L(\Omega),
$$

thus (20) holds and (ii) is proved. This achieves the proof of Lemma 1.

\section{Proof of Theorem 2}

\subsection{Proof of point (i) of the theorem}

Given a sequence $\left(\varepsilon_{n}\right)_{n \geq 1}$ with $\varepsilon_{n} \downarrow 0$ as $n$ goes to infinity, we first consider a sequence $\left(v_{n}, u_{n}\right)_{n \geq 1}$ that satisfies $(12)$.

Then, by standard results $[15,1]$ on the Cahn-Hilliard energy $L_{\varepsilon}$, we know that up to a subsequence (still denoted by $v_{n}$ ), there exists a Caccioppoli set $F \subseteq Q^{+}$such that $v_{n} \rightarrow \chi_{F}$ a.e. in $Q^{+}$, moreover,

$$
\sigma_{c} \mathcal{H}^{1}\left(\partial^{*} F\right) \leq \liminf _{n \rightarrow \infty} L_{\varepsilon_{n}}\left(v_{n}\right)
$$

where $\partial^{*} F$ is the reduced boundary of $F$ inside $Q^{+}$. Since $\sup _{n \geq 1} \int_{Q^{+}} v_{n}(\xi) d \xi<+\infty$ the set $F$ is bounded, and since $\partial_{y} v_{n} \leq 0, F \cup \overline{Q^{-}}$is Lebesgue-essentially equivalent to the sub-graph of a (non-negative) bounded variation function $g: \mathbf{S}^{1} \rightarrow[0,+\infty)$. 
Now fix an integer $k \geq 2$ and write for every $n$, using the co-area formula (see (54), Appendix A)

$$
\begin{aligned}
f_{\frac{1}{k+1}}^{\frac{1}{k}}\left|D \chi_{\left\{v_{n}>s\right\}}\right|\left(Q^{+}\right) d s & =k(k+1) \int_{\left\{\xi \in Q^{+}: \frac{1}{k+1}<v_{n}(\xi)<\frac{1}{k}\right\}}\left|\nabla v_{n}(\xi)\right| d \xi \\
& \leq(k(k+1))^{\frac{3}{2}} \int_{Q^{+}}\left|\nabla v_{n}(\xi)\right| \sqrt{v_{n}(\xi)\left(1-v_{n}(\xi)\right)} d \xi \\
& \leq(k(k+1))^{\frac{3}{2}} \frac{\pi}{8 \sigma_{c}} L_{\varepsilon_{n}}\left(v_{n}\right)
\end{aligned}
$$

which by (12) is uniformly bounded by some constant $c_{k}$. We deduce that there exist a level $s_{n}^{k} \in\left(\frac{1}{k+1}, \frac{1}{k}\right)$ such that $\left|D \chi_{\left\{v_{n}>s_{n}^{k}\right\}}\right|\left(Q^{+}\right) \leq c_{k}$.

Moreover, since $\partial_{y} v_{n} \leq 0,\left\{v_{n}>s_{n}^{k}\right\}$ is the sub-graph of some function of bounded variation. Thus, if we define $h_{n}^{k}: \mathbf{S}^{1} \rightarrow[0,+\infty)$ to be the largest l.s.c. representative of this function, the open set $\Omega_{n}^{k}=\left\{(x, y) \in Q: y<h_{n}^{k}(x)\right\} \in \mathcal{G}$ is such that $\frac{\circ}{\Omega_{n}^{k}}=\Omega_{n}^{k}$ and is (Lebesgue-) essentially equal in $Q^{+}$to $\left\{v_{n}>s_{n}^{k}\right\}$. In particular, $v_{n}>s_{n}^{k}$ a.e. in $\Omega_{n}^{k}, v_{n} \leq s_{n}^{k}$ a.e. out of $\Omega_{n}^{k}$, and $\mathcal{H}^{1}\left(\partial \Omega_{n}^{k} \cap Q^{+}\right)=\left|D \chi_{\left\{v_{n}>s_{n}^{k}\right\}}\right|\left(Q^{+}\right) \leq c_{k}$ so that $\mathcal{H}^{1}\left(\partial \Omega_{n}^{k}\right) \leq 1+c_{k}$.

Define the sequence $\left(n_{p}^{1}\right)_{p \geq 1}$ by $n_{p}^{1}=p$ for every $p$. For each $k=2,3, \ldots$, we can extract (by induction) from the sequence $\left(n_{p}^{k-1}\right)$ a sequence $\left(n_{p}^{k}\right)$ such that $\Omega_{n_{p}^{k}}^{k}$ converges to some open set $\Omega^{k} \in \mathcal{G}$ as $p$ goes to infinity. We build in this way a family of sets $\left(\Omega^{k}\right)_{k \geq 2}$ such that for every $k$, the sequence $\left(\Omega_{n_{p}^{p}}^{k}\right)$ converges to $\Omega^{k}$ in $\mathcal{G}$.

In the sequel we will relabel this subsequence and denote again by $n$ what should be $n_{p}^{p}$.

We let $\Omega=\bigcup_{k \geq 2} \Omega^{k}$ and

$$
\begin{aligned}
N= & \left\{\xi \in Q^{+}: v_{n}(\xi) \nrightarrow \chi_{F}(\xi) \text { as } n \rightarrow \infty\right\} \\
& \cup \bigcup_{k, n}\left(\left\{v_{n}>s_{n}^{k}\right\} \backslash \Omega_{n}^{k}\right) \cup\left(\left\{v_{n} \leq s_{n}^{k}\right\} \cap \Omega_{n}^{k}\right),
\end{aligned}
$$

and observe that $|N|=0$. Notice that $\Omega$ has the following characterization:

$$
Q \backslash \Omega=\left\{\xi \in Q: \forall \rho>0, \lim _{n \rightarrow \infty} \inf \operatorname{ess}_{B(\xi, \rho)} v_{n}=0\right\}
$$

Indeed, consider $\xi \notin \Omega$, and fix $\rho>0$. For any fixed $k \geq 2$, there exists $\xi_{n} \notin \Omega_{n}^{k}$ such that $\xi_{n} \rightarrow \xi$. But since $|N|=0$ and $\Omega_{n}^{k}=\frac{\circ}{\Omega_{n}^{k}}$, we have that $\left|B\left(\xi_{n}, \rho / 2\right) \backslash \Omega_{n}^{k} \backslash N\right|>0$. If $n$ is large enough, $\left|\xi_{n}-\xi\right|<\rho / 2$, therefore $B(\xi, \rho) \supset B\left(\xi_{n}, \rho / 2\right)$ and $\inf \operatorname{ess}_{B(\xi, \rho)} v_{n} \leq$ $s_{n}^{k}<1 / k$. This shows that for every $k \geq 2$, $\limsup _{n \rightarrow \infty} \inf \operatorname{ess}_{B(\xi, \rho)} v_{n} \leq 1 / k$ : thus $\lim _{n \rightarrow \infty} \inf _{\operatorname{ess}}(\xi, \rho) v_{n}=0$. We will not need it in the sequel, but it is also easy to show that if $\xi \in \Omega$, then $\lim \sup _{n \rightarrow \infty} \inf \operatorname{ess}_{B(\xi, \rho)} v_{n}>0$. 
We will now prove that the set $\Omega$ satisfies the thesis of point (i) of Theorem 2. We will first show that

$$
L(\Omega) \leq \liminf _{n \rightarrow \infty} L_{\varepsilon_{n}}\left(v_{n}\right),
$$

in section 6.1.1 and then, in section 6.1.2, we will show that (up to a subsequence) $u_{n}$ converges to a function $u$ such that

$$
\begin{aligned}
\int_{\Omega \cap Q^{+}} \mathbf{A} e(u)(x, y): e(u)(x, y) d x d y \\
\quad \leq \liminf _{n \rightarrow \infty} \int_{Q^{+}}\left(v_{n}(x, y)+\eta_{\varepsilon_{n}}\right) \mathbf{A} e\left(u_{n}\right)(x, y): e\left(u_{n}\right)(x, y) d x d y
\end{aligned}
$$

\subsubsection{Estimate of $L(\Omega)$}

In order to show (35) we need more information on the structure of $\partial \Omega$ and on the relationship of $\Omega$ with $F$.

Notice that if $\xi \in \Omega \backslash N \cap Q^{+}, \xi \in \Omega^{k}$ for some $k$, and therefore $\xi \in \Omega_{n}^{k}$ for large $n$. In particular, $v_{n}(\xi)>s_{n}^{k}>1 /(k+1)$ and since it tends either to 0 or to 1 , $\lim _{n \rightarrow \infty} v_{n}(\xi)=1$ and $\xi \in F$. Therefore $\Omega \cap Q^{+} \backslash N \subseteq F$.

On the other hand, we claim that $|F \backslash \Omega|=0$.

Indeed, we first notice that, given any $k \geq 2, \chi_{\Omega_{n}^{k}} \rightarrow \chi_{\Omega^{k}}$ a.e. in $Q^{+}$as $n \rightarrow \infty$ (up to a subsequence) and thus, for instance, in $L^{2}\left(Q^{a}\right)$ for every $a>0$. This is true because up to a subsequence, we can assume that $\partial \Omega_{n}^{k}$ tends to some compact subset $K \subset \overline{Q^{+}}$in the Hausdorff sense: then, by Golab's theorem (see [12, 8, 16]) $\mathcal{H}^{1}(K) \leq \liminf _{n \rightarrow \infty} \mathcal{H}^{1}\left(\partial \Omega_{n}^{k}\right)<+\infty\left(\right.$ since $\mathcal{H}^{1}\left(\partial \Omega_{n}^{k}\right) \leq 1+c_{k}$ for every $\left.n\right)$ and thus $|K|=0$. Eventually, observe that if $\xi \in \Omega^{k}$, then $\xi \in \Omega_{n}^{k}$ for large $n$ and $\chi_{\Omega_{n}^{k}}(\xi)=1$, and that if $\xi \notin \Omega^{k} \cup K$, we can easily show that $\xi \notin \Omega_{n}^{k}$ for large $n$ and $\chi_{\Omega_{n}^{k}}(\xi)=0$ (see section 5.1 for details of a similar proof).

Then, we have $v_{n} \rightarrow 1$ a.e. in $F$, and in particular $v_{n} \chi_{F} \rightarrow \chi_{F}$ in $L^{2}\left(Q^{+}\right)$. Thus,

$$
\begin{aligned}
\left|Q^{a} \cap F \backslash \Omega^{k}\right| & =\int_{Q^{a}} \chi_{F}(\xi)\left(1-\chi_{\Omega^{k}}\right)(\xi) d \xi \\
& =\lim _{n \rightarrow \infty} \int_{Q^{a}} v_{n}(\xi) \chi_{F}(\xi)\left(1-\chi_{\Omega_{n}^{k}}\right)(\xi) d \xi \\
& =\lim _{n \rightarrow \infty} \int_{Q^{a}} v_{n}(\xi) \chi_{F}(\xi) \chi_{\left\{v_{n} \leq s_{n}^{k}\right\}}(\xi) d \xi \\
& \leq \lim _{n \rightarrow \infty} \frac{1}{k} \int_{Q^{a}} \chi_{F}(\xi)\left(1-\chi_{\Omega_{n}^{k}}\right)(\xi) d \xi=\frac{1}{k}\left|Q^{a} \cap F \backslash \Omega^{k}\right|,
\end{aligned}
$$

so that $\left|Q^{a} \cap F \backslash \Omega^{k}\right|$ must be zero for any $a>0$. This shows that $\left|F \backslash \Omega^{k}\right|=0$, and since $\Omega^{k} \subset \Omega$ it proves the claim. We conclude that $\Omega \cap Q^{+}=F$ up to a Lebesgue-negligible set.

Recall that $F$ is the sub-graph of a function of bounded variation $g \in B V\left(\mathbf{S}^{1}\right)$. Let $\bar{h}$ be the greatest 1.s.c. representative of $g(\bar{h}$ can be built, for instance, as the l.s.c. 
envelope of any upper-semicontinuous representative of $g$ ). Since $\Omega \cap Q^{+}=F$ up to a Lebesgue-negligible set, we easily show that

$$
\stackrel{\circ}{\Omega}=\{(x, y) \in Q: y<\bar{h}(x)\},
$$

and that $\partial \bar{\Omega} \cap Q^{+}$is $\mathcal{H}^{1}$-essentially equal to $\partial^{*} F$.

For any Borel set $B \subseteq Q$ we introduce the following localization of $L_{\varepsilon}$

$$
L_{\varepsilon}(v, B)=2 \sigma_{c}\left(\frac{4 \varepsilon}{\pi^{2}} \int_{Q^{+} \cap B}|\nabla v(x, y)|^{2} d x d y+\frac{1}{\varepsilon} \int_{Q^{+} \cap B} v(x, y)(1-v(x, y)) d x d y\right) .
$$

For any open set $A$, we also have $\sigma_{c} \mathcal{H}^{1}\left(\partial^{*} F \cap A\right) \leq \liminf _{n \rightarrow \infty} L_{\varepsilon_{n}}\left(v_{n}, A\right)$. In particular, if we choose $\delta>0$, and define $A^{\delta}=\left\{\xi \in Q: \operatorname{dist}\left(\xi, \partial \bar{\Omega} \cap Q^{+}\right)<\delta\right\}$, we have that

$$
\sigma_{c} \mathcal{H}^{1}\left(\partial \bar{\Omega} \cap Q^{+}\right) \leq \liminf _{n \rightarrow \infty} L_{\varepsilon_{n}}\left(v_{n}, A^{\delta}\right) .
$$

Define now $B^{\delta}=\left\{\xi \in Q \backslash \overline{A^{\delta}}: \operatorname{dist}\left(\xi, \partial \bar{\Omega} \backslash Q^{+}\right)<\delta\right\}: B^{\delta} \cap \partial \bar{\Omega}$ is a finite union of segments in $\mathbf{S}^{1}$. We want to estimate $\mathcal{H}^{1}\left(\partial \bar{\Omega} \cap B^{\delta}\right)$. Write $\partial \bar{\Omega} \cap B^{\delta}=\bigcup_{k=1}^{N}\left(a_{k}, b_{k}\right) \times\{0\}$. We have for any $\delta^{\prime} \leq \delta$

$$
\begin{aligned}
L_{\varepsilon_{n}}\left(v_{n}, B^{\delta}\right) & \geq \sum_{k=1}^{N} \int_{a_{k}}^{b_{k}}\left\{2 \sigma_{c} \int_{0}^{\delta^{\prime}} \frac{4 \varepsilon_{n}}{\pi^{2}}\left|\partial_{y} v_{n}(x, y)\right|^{2}+\frac{v_{n}(x, y)\left(1-v_{n}(x, y)\right)}{\varepsilon_{n}} d y\right\} d x \\
& \geq \sum_{k=1}^{N} \int_{a_{k}}^{b_{k}}\left\{\frac{8 \sigma_{c}}{\pi} \int_{0}^{\delta^{\prime}} \sqrt{v_{n}(x, y)\left(1-v_{n}(x, y)\right)}\left|\partial_{y} v_{n}(x, y)\right| d y\right\} d x .
\end{aligned}
$$

Recalling that $v_{n}(x, 0)=v_{s}$, where $v_{s}$ is defined by (11), we deduce

$$
\begin{aligned}
L_{\varepsilon_{n}}\left(v_{n}, B^{\delta}\right) & \geq \sum_{k=1}^{N} \int_{a_{k}}^{b_{k}}\left\{\frac{8 \sigma_{c}}{\pi} \int_{v_{n}\left(x, \delta^{\prime}\right)}^{v_{s}} \sqrt{t(1-t)} d t\right\} d x \\
& =\sum_{k=1}^{N} \int_{a_{k}}^{b_{k}}\left\{\sigma_{c} \wedge \sigma_{s}-\frac{8 \sigma_{c}}{\pi} \int_{0}^{v_{n}\left(x, \delta^{\prime}\right)} \sqrt{t(1-t)} d t\right\} d x .
\end{aligned}
$$

Since $v_{n} \rightarrow 0$ a.e. in $B^{\delta} \cap Q^{+}$, for a.e. $\delta^{\prime} \in(0, \delta]$, we must have that for a.e. $x$ in $\bigcup_{k=1}^{N}\left(a_{k}, b_{k}\right), v_{n}\left(x, \delta^{\prime}\right) \rightarrow 0$ as $n \rightarrow \infty$. Therefore, choosing such a $\delta^{\prime}$, we have for a.e. $x \in \bigcup_{k=1}^{N}\left(a_{k}, b_{k}\right)$,

$$
\lim _{n \rightarrow \infty} \int_{0}^{v_{n}\left(x, \delta^{\prime}\right)} \sqrt{t(1-t)} d t=0
$$

so that

$$
\liminf _{n \rightarrow \infty} L_{\varepsilon_{n}}\left(v_{n}, B^{\delta}\right) \geq\left(\sigma_{c} \wedge \sigma_{s}\right) \sum_{k=1}^{N}\left|b_{k}-a_{k}\right|=\left(\sigma_{c} \wedge \sigma_{s}\right) \mathcal{H}^{1}\left(\partial \bar{\Omega} \cap B^{\delta}\right) .
$$


Now, we want to estimate the length of $\partial \Omega \backslash \partial \bar{\Omega}$. As usual $h: \mathbf{S}^{1} \rightarrow[0,+\infty)$ will denote the 1.s.c. function such that $\Omega=\{(x, y) \in Q: y<h(x)\}$, and it has been shown in section 5.1 that

$$
\partial \Omega \backslash \partial \bar{\Omega}=\bigcup_{x \in \mathbf{S}^{1}}\{x\} \times[h(x), \bar{h}(x)) .
$$

Given $\delta>0$, choose $x_{0} \in \mathbf{S}^{1}$ such that $\bar{h}\left(x_{0}\right)-h\left(x_{0}\right)>\delta$ and $\eta>0$, small enough to have (since $\left.\left[h\left(x_{0}\right), \bar{h}\left(x_{0}\right)-\delta\right] \subset \stackrel{\circ}{\Omega}\right)$

$$
C^{\delta, \eta}=\left[x_{0}-\eta, x_{0}+\eta\right] \times\left[h\left(x_{0}\right), \bar{h}\left(x_{0}\right)-\delta\right] \subset \bar{\Omega}
$$

Let $\rho>0, \rho<\min \{\delta, \eta\} / 2$. Since $\left(x_{0}, h\left(x_{0}\right)\right) \notin \Omega$ and $\Omega$ is characterized by (34), $\lim _{n \rightarrow \infty} \inf _{\operatorname{ess}_{B}\left(\left(x_{0}, h\left(x_{0}\right)\right), \rho\right)} v_{n}=0$. As $v_{n}$ is nonincreasing in $y$, there exists $x_{n} \in$ $\left(x_{0}-\rho, x_{0}+\rho\right)$ such that $v_{n}\left(x_{n}, y\right) \rightarrow 0$ as $n \rightarrow \infty$ for a.e. $y \in\left(h\left(x_{0}\right)+\rho,+\infty\right)$. On the other hand, $v_{n} \rightarrow 1$ a.e. in $\bar{\Omega}$, thus a.e. in $\left(x_{0}-\eta, x_{0}-\rho\right) \times\left(\bar{h}\left(x_{0}\right)-\delta-\rho, \bar{h}\left(x_{0}\right)-\delta\right)$ and in $\left(x_{0}+\rho, x_{0}+\eta\right) \times\left(\bar{h}\left(x_{0}\right)-\delta-\rho, \bar{h}\left(x_{0}\right)-\delta\right)$, and there exist $x_{n}^{\prime} \in\left(x_{0}-\eta, x_{0}-\rho\right)$, $x_{n}^{\prime \prime} \in\left(x_{0}+\rho, x_{0}+\eta\right)$ such that $v_{n}\left(x_{n}^{\prime}, y\right)$ and $v_{n}\left(x_{n}^{\prime \prime}, y\right)$ converge to 1 as $n \rightarrow \infty$ for a.e. $y \in\left(0, h\left(x_{0}\right)-\delta-\rho\right)$.

Now, we have $L_{\varepsilon_{n}}\left(v_{n}, C^{\delta, \eta}\right) \geq$

$$
\begin{aligned}
& \geq 2 \sigma_{c} \int_{h\left(x_{0}\right)+\rho}^{\bar{h}\left(x_{0}\right)-\delta-\rho}\left\{\int_{x_{n}^{\prime}}^{x_{n}^{\prime \prime}} \frac{4 \varepsilon_{n}}{\pi^{2}}\left|\partial_{x} v_{n}(x, y)\right|^{2}+\frac{v_{n}(x, y)\left(1-v_{n}(x, y)\right)}{\varepsilon_{n}} d x\right\} d y \\
& \geq \frac{8 \sigma_{c}}{\pi} \int_{h\left(x_{0}\right)+\rho}^{\bar{h}\left(x_{0}\right)-\delta-\rho}\left\{-\int_{x_{n}^{\prime}}^{x_{n}} \sqrt{v_{n}\left(1-v_{n}\right)} \partial_{x} v_{n} d x+\int_{x_{n}}^{x_{n}^{\prime \prime}} \sqrt{v_{n}\left(1-v_{n}\right)} \partial_{x} v_{n} d x\right\} d y \\
& =\frac{8 \sigma_{c}}{\pi} \int_{h\left(x_{0}\right)+\rho}^{\bar{h}\left(x_{0}\right)-\delta-\rho}\left\{\int_{v_{n}\left(x_{n}, y\right)}^{v_{n}\left(x_{n}^{\prime \prime}, y\right)} \sqrt{t(1-t)} d t+\int_{v_{n}\left(x_{n}, y\right)}^{v_{n}\left(x_{n}^{\prime}, y\right)} \sqrt{t(1-t)} d t\right\} d y .
\end{aligned}
$$

Since for a.e. $y \in\left(h\left(x_{0}\right)+\rho, \bar{h}\left(x_{0}\right)-\delta-\rho\right)$, the terms $\int_{v_{n}\left(x_{n}, y\right)}^{v_{n}\left(x_{n}^{\prime \prime}, y\right)} \sqrt{t(1-t)} d t$ and $\int_{v_{n}\left(x_{n}, y\right)}^{v_{n}\left(x_{n}^{\prime}, y\right)} \sqrt{t(1-t)} d t$ converge to $\int_{0}^{1} \sqrt{t(1-t)} d t=\pi / 8$ as $n \rightarrow \infty$, we get

$$
\liminf _{n \rightarrow \infty} L_{\varepsilon_{n}}\left(v_{n}, C^{\delta, \eta}\right) \geq 2 \sigma_{c}\left(\bar{h}\left(x_{0}\right)-h\left(x_{0}\right)-\delta-2 \rho\right),
$$

and sending $\rho$ to zero,

$$
2 \sigma_{c}\left(\bar{h}\left(x_{0}\right)-h\left(x_{0}\right)-\delta\right) \leq \liminf _{n \rightarrow \infty} L_{\varepsilon_{n}}\left(v_{n}, C^{\delta, \eta}\right) .
$$

We now are able to show (35). Choose $x_{1}, \ldots, x_{N}$ such that $h\left(x_{k}\right)<\bar{h}\left(x_{k}\right)$ and $\delta<\min _{k=1, \ldots, N}\left(\bar{h}\left(x_{k}\right)-h\left(x_{k}\right)\right)$, and choose $\eta>0$ such that the sets $C_{k}^{\delta, \eta}=\left[x_{k}-\right.$ $\left.\eta, x_{k}+\eta\right] \times\left[h\left(x_{k}\right), \bar{h}\left(x_{k}\right)-\delta\right]$ are disjoint and all included in $\stackrel{\circ}{\Omega}$. Choose also $\delta^{\prime}>0$ 
such that the (disjoint) sets $A^{\delta^{\prime}}, B^{\delta^{\prime}}$ defined as before do not touch any of the $C_{k}^{\delta, \eta}$. From (38), (39) and (40), we get that

$$
\begin{aligned}
\liminf _{n \rightarrow \infty} L_{\varepsilon_{n}}\left(v_{n}\right) \geq \liminf _{n \rightarrow \infty} L_{\varepsilon_{n}}\left(v_{n}, A^{\delta^{\prime}}\right) & +\liminf _{n \rightarrow \infty} L_{\varepsilon_{n}}\left(v_{n}, B^{\delta^{\prime}}\right) \\
& +\sum_{k=1}^{N} \liminf _{n \rightarrow \infty} L_{\varepsilon_{n}}\left(v_{n}, C_{k}^{\delta, \eta}\right) \\
\geq \sigma_{c} \mathcal{H}^{1}\left(\partial \bar{\Omega} \cap Q^{+}\right) & +\left(\sigma_{c} \wedge \sigma_{s}\right) \mathcal{H}^{1}\left(\partial \bar{\Omega} \cap B^{\delta^{\prime}}\right) \\
& +2 \sigma_{c} \sum_{k=1}^{N}\left(\bar{h}\left(x_{k}\right)-h\left(x_{k}\right)-\delta\right)
\end{aligned}
$$

Sending first $\delta^{\prime}$ to zero, and then $\delta$, we get

$$
\sigma_{c} \mathcal{H}^{1}\left(\partial \bar{\Omega} \cap Q^{+}\right)+\left(\sigma_{c} \wedge \sigma_{s}\right) \mathcal{H}^{1}(\partial \bar{\Omega})+2 \sigma_{c} \sum_{k=1}^{N}\left(\bar{h}\left(x_{k}\right)-h\left(x_{k}\right)\right) \leq \liminf _{n \rightarrow \infty} L_{\varepsilon_{n}}\left(v_{n}\right)
$$

Since this is true for any $\left\{x_{1}, \ldots, x_{N}\right\} \subseteq\left\{x \in \mathbf{S}^{1}: h(x)<\bar{h}(x)\right\}$, we deduce (35).

\subsubsection{Convergence of the sequence $\left(u_{n}\right)_{n \geq 1}$}

First of all, (since (12) holds) we may extract a subsequence (still denoted by $\left(u_{n}, v_{n}\right)$ ) such that $\sqrt{v_{n}} e\left(u_{n}\right)$ weakly converges in $L^{2}\left(Q^{+} ; \mathbf{R}^{4}\right)$ to some function $\mathcal{E}$.

Consider now a Lipschitz sub-graph $A \subset \Omega$, with $A \cap Q^{+} \subset \subset \Omega$. Since $\overline{A \cap Q^{+}}$ is compact and included in $\bigcup_{k \geq 2} \Omega^{k}$, there exists $k \geq 2$ such that $A \cap Q^{+} \subset \subset \Omega^{k}$, in particular, $A \subset \Omega_{n}^{k}$ for large $n$. Since $v_{n}>s_{n}^{k}>1 /(k+1)$ a.e. in $\Omega_{n}^{k}, 1 / \sqrt{v_{n}}$ is uniformly bounded by $\sqrt{k+1}$ in $A$ and since it converges to 1 a.e., it follows that $e\left(u_{n}\right)=\sqrt{v_{n}} e\left(u_{n}\right) \times 1 / \sqrt{v_{n}} \rightarrow \mathcal{E}$ weakly in $L^{2}\left(A \cap Q^{+} ; \mathbf{R}^{4}\right)$ as $n \rightarrow \infty$. Using Korn's inequality and the fact that $e\left(u_{n}\right)$ is uniformly bounded in $L^{2}\left(A \cap Q^{+} ; \mathbf{R}^{4}\right)$, we deduce (since $u_{n}-(x, 0) \equiv 0$ on $\left.\{y=0\}\right)$ that $\left(u_{n}\right)_{n \geq 1}$ is also compact in $L_{l o c}^{2}\left(\pi^{-1}\left(A \cap Q^{+}\right) ; \mathbf{R}^{2}\right)$, and since if $u$ is a limit point of the sequence we must have $u-(x, 0) \equiv 0$ on $\{y=0\}$ and $e(u)=\mathcal{E}$ the possible limit point is unique, therefore $u_{n}$ converges in $L^{2}\left(A \cap Q^{+} ; \mathbf{R}^{2}\right)$.

Since this holds for every Lipschitz sub-graph $A$ with $A \cap Q^{+} \subset \subset \Omega, u_{n}$ converges in $L_{l o c}^{2}\left(\pi^{-1}\left(\Omega \cap Q^{+}\right) ; \mathbf{R}^{2}\right)$ to some function $u \in X(\Omega)$, with $e(u)=\mathcal{E}$ in the distributional sense. As $\mathcal{E}$ is the weak limit in $L^{2}\left(Q^{+} ; \mathbf{R}^{4}\right)$ of $\sqrt{v_{n}} e\left(u_{n}\right),(36)$ follows and point (i) of Theorem (2) is proved.

It remains to prove (ii).

\subsection{Proof of point (ii) of Theorem 2}

\subsubsection{The optimal profile for $L_{\varepsilon}$}

Consider the following 1-dimensional version of $L_{\varepsilon}$

$$
l_{\varepsilon}(v, I)=2 \sigma_{c}\left(\frac{4 \varepsilon}{\pi^{2}} \int_{I}\left|v^{\prime}(t)\right|^{2} d t+\frac{1}{\varepsilon} \int_{I} v(t)(1-v(t)) d t\right),
$$


defined for and interval $I \subseteq \mathbf{R}$ and $v: I \rightarrow[0,1]$. Consider also the two problems

$$
\min \left\{l_{\varepsilon}(v, \mathbf{R}): v: \mathbf{R} \rightarrow[0,1], v^{\prime} \leq 0, \lim _{-\infty} v=1, \lim _{+\infty} v=0\right\}
$$

and

$$
\min \left\{l_{\varepsilon}(v,[0,+\infty)): v:[0,+\infty) \rightarrow[0,1], v^{\prime} \leq 0, v(0)=v_{s}, \lim _{+\infty} v=0\right\} .
$$

Is is known (and easy to prove) that problem (42) is solved by $v_{\varepsilon}(t)=\gamma\left(\frac{t}{\varepsilon}\right)$, where the optimal profile $\gamma$ is

$$
\gamma(t)= \begin{cases}1 & \text { if } t \leq-1 \\ \frac{1}{2}\left(1-\sin \frac{\pi t}{2}\right) & \text { if }-1 \leq t \leq 1 \\ 0 & \text { if } t \geq 1\end{cases}
$$

The value of the minimum (42) is $\sigma_{c}$. Similarly, problem (43) is solved by the function $\tilde{v}_{\varepsilon}(t)=\tilde{\gamma}\left(\frac{t}{\varepsilon}\right)$, where for any $t \geq 0, \tilde{\gamma}(t)=\gamma\left(t+t_{s}\right)$ and $t_{s}=\frac{2}{\pi} \arcsin \left(1-2 v_{s}\right) \in[-1,1)$, so that $\gamma\left(t_{s}\right)=v_{s}$. In this case the value of the minimum (43) is $\sigma_{c} \wedge \sigma_{s}$.

\subsubsection{Construction of a sequence $\left(v_{\varepsilon}, u_{\varepsilon}\right)$, for a regular $(\Omega, u)$}

We will first consider the case where $\partial \Omega$ is regular: we assume that $\Omega$ is the sub-graph of a Lipschitz function $h$, that $\partial \Omega \cap Q^{+}$is a finite union of $C^{2}$ arcs, and that $\partial \Omega \backslash Q^{+}$ is a finite union of segments $\left(a_{k}, b_{k}\right) \times\{0\} \subset \mathbf{S}^{1} \times\{0\}, k=1, \ldots, N$. We also assume that $u \in L^{\infty}\left(\Omega, \mathbf{R}^{2}\right)$.

We define the signed distance $d_{\Omega}$ to $\partial \Omega$ as

$$
d_{\Omega}(\xi)=\operatorname{dist}(\xi, \Omega)-\operatorname{dist}(\xi, Q \backslash \Omega) .
$$

In particular, $d_{\Omega}(\xi)=0 \Leftrightarrow \xi \in \partial \Omega$ and $d_{\Omega}(x, y) \leq y<0$ if $y<0$.

For every $\varepsilon>0$ (small) and $\xi \in Q$ we define

$$
v_{\varepsilon}(\xi)=\gamma\left(\frac{d_{\Omega}(\xi)}{\varepsilon}+t_{s}\right)
$$

so that $v_{\varepsilon} \equiv v_{s}$ on $\partial \Omega$, and, in particular, $v_{\varepsilon} \geq v_{s}$ on $\partial Q^{+}=\mathbf{S}^{1} \times\{0\}$. Notice that $\left|\nabla v_{\varepsilon}(\xi)\right|=\left|\gamma^{\prime}\left(d_{\Omega}(\xi) / \varepsilon+t_{s}\right) \nabla d_{\Omega}(\xi) / \varepsilon\right|=-\gamma^{\prime}\left(d_{\Omega}(\xi) / \varepsilon+t_{s}\right) / \varepsilon$ a.e. in $Q$, and that $\left|\nabla v_{\varepsilon}(\xi)\right|=v_{\varepsilon}(\xi)\left(1-v_{\varepsilon}(\xi)\right)=0$ if $\left|d_{\Omega}(\xi)\right|>2 \varepsilon$.

If $A \subseteq Q$ is open, $\left(L_{\varepsilon}\left(v_{\varepsilon}, A\right)\right.$ defined by (37)), is bounded by

$$
\begin{aligned}
L_{\varepsilon}\left(v_{\varepsilon}, A\right) & \leq \\
& \leq \frac{2 \sigma_{c}}{\varepsilon} \int_{Q^{+} \cap A} \frac{4}{\pi^{2}} \gamma^{\prime}\left(\frac{d_{\Omega}(\xi)}{\varepsilon}+t_{s}\right)^{2}+\gamma\left(\frac{d_{\Omega}(\xi)}{\varepsilon}+t_{s}\right)\left(1-\gamma\left(\frac{d_{\Omega}(\xi)}{\varepsilon}+t_{s}\right)\right) d \xi \\
& \leq 4 \sigma_{c} \frac{1}{4 \varepsilon}\left|\left\{\xi \in A:\left|d_{\Omega}(\xi)\right|<2 \varepsilon\right\}\right| \\
& \leq 4 \sigma_{c} \mathcal{H}^{1}(\partial \Omega \cap A)+c_{\varepsilon},
\end{aligned}
$$


with $c_{\varepsilon}=c_{\varepsilon}(A, \delta)$ converging to 0 as $\varepsilon \rightarrow 0$, since, as $\partial \Omega$ is closed, the Minkowski content $\lim _{\varepsilon \downarrow 0} \frac{1}{2 \varepsilon}\left|\left\{\xi \in A:\left|d_{\Omega}(\xi)\right|<\varepsilon\right\}\right|$ is equal to the length $\mathcal{H}^{1}(\partial \Omega \cap A)$ (see for instance [10]).

Now, given $\delta>0$ small, we let $\partial \Omega_{+}^{\delta}=\left\{\xi \in \partial \Omega \cap Q^{+}: \operatorname{dist}\left(\xi, Q^{-}\right)>\delta\right\}, \partial \Omega_{0}^{\delta}=$ $\left\{\xi \in \partial \Omega \backslash Q^{+}: \operatorname{dist}\left(\xi, \partial \Omega \cap Q^{+}\right)>\delta\right\}$, and $A_{+}^{\delta}=\left\{\xi+\zeta \nu(\xi): \xi \in \partial \Omega_{+}^{\delta},-\delta<\zeta<\delta\right\}$, where $\nu(\xi)$ is the normal to $\partial \Omega$ at $\xi, A_{0}^{\delta}=\left\{\xi+(0, \zeta): \xi \in \partial \Omega_{0}^{\delta}, 0<\zeta<\delta\right\}$. Notice that $A_{+}^{\delta} \cup A_{0}^{\delta} \subset Q^{+}$. We let $B^{\delta}=\left\{\xi \in Q: \operatorname{dist}\left(\xi, \partial \Omega \backslash\left(\partial \Omega_{+}^{\delta} \cup \partial \Omega_{0}^{\delta}\right)\right)<2 \delta\right\}$.

If $\delta$ is small enough, $\left\{\xi \in Q^{+}: \operatorname{dist}(\xi, \partial \Omega)<\delta\right\} \subset A_{0}^{\delta} \cup A_{+}^{\delta} \cup B^{\delta}$, and if $2 \varepsilon<\delta$,

$$
L_{\varepsilon}\left(v_{\varepsilon}\right) \leq L_{\varepsilon}\left(v_{\varepsilon}, A_{0}^{\delta}\right)+L_{\varepsilon}\left(v_{\varepsilon}, A_{+}^{\delta}\right)+L_{\varepsilon}\left(v_{\varepsilon}, B^{\delta}\right) .
$$

The last quantity is

$$
L_{\varepsilon}\left(v_{\varepsilon}, B^{\delta}\right) \leq 4 \sigma_{c} \mathcal{H}^{1}\left(\partial \Omega \cap B^{\delta}\right)+c_{\varepsilon}
$$

by (45). It is easy to check that

$$
\partial \Omega \cap B^{\delta} \subset C^{\delta}=\left(\partial \Omega \cap Q^{3 \delta}\right) \cup\left(\left(\partial \Omega \backslash Q^{+}\right) \backslash d o m_{0}^{3 \delta}\right),
$$

so that the previous inequality yields

$$
L_{\varepsilon}\left(v_{\varepsilon}, B^{\delta}\right) \leq 4 \sigma_{c} \mathcal{H}^{1}\left(C^{\delta}\right)+c_{\varepsilon} .
$$

We estimate the two other integrals:

$$
\begin{aligned}
L_{\varepsilon}\left(v_{\varepsilon},\right. & \left.A_{0}^{\delta}\right)={ }_{k=1}^{N} \int_{a_{k}+\delta}^{b_{k}-\delta}\left(\frac{2 \sigma_{c}}{\varepsilon} \int_{0}^{\delta} \frac{4}{\pi^{2}} \gamma^{\prime}\left(\frac{\zeta}{\varepsilon}+t_{s}\right)^{2}+\gamma\left(\frac{\zeta}{\varepsilon}+t_{s}\right)\left(1-\gamma\left(\frac{\zeta}{\varepsilon}+t_{s}\right)\right) d \zeta\right) d x \\
& =\left(\sum_{k=1}^{N}\left|b_{k}-a_{k}\right|-2 N \delta\right) 2 \sigma_{c} \int_{t_{s}}^{\delta / \varepsilon} \frac{4}{\pi^{2}} \gamma^{\prime}(t)^{2}+\gamma(t)(1-\gamma(t)) d t \\
& \leq\left(\sigma_{c} \wedge \sigma_{s}\right)\left(\mathcal{H}^{1}\left(\partial \Omega \backslash Q^{+}\right)-2 N \delta\right)
\end{aligned}
$$

by definition of $t_{s}$. On the other hand,

$$
\begin{aligned}
L_{\varepsilon}\left(v_{\varepsilon}, A_{+}^{\delta}\right)= \\
\quad=\int_{\partial \Omega_{+}^{\delta}} d \mathcal{H}^{1}(z)\left(\frac{2 \sigma_{c}}{\varepsilon} \int_{-\delta}^{\delta} J(z, \zeta) \frac{4}{\pi^{2}} \gamma^{\prime}\left(\frac{\zeta}{\varepsilon}+t_{s}\right)^{2}+\gamma\left(\frac{\zeta}{\varepsilon}+t_{s}\right)\left(1-\gamma\left(\frac{\zeta}{\varepsilon}+t_{s}\right)\right) d \zeta\right) \\
\quad=\int_{\partial \Omega_{+}^{\delta}} d \mathcal{H}^{1}(z)\left(2 \sigma_{c} \int_{-1}^{1} J\left(z, \varepsilon\left(t-t_{s}\right)\right) \frac{1}{2} \cos ^{2}\left(\frac{\pi t}{2}\right) d t\right),
\end{aligned}
$$

where $J(z, \zeta)$ is the Jacobian of the transformation $\xi \in A_{+}^{\delta} \mapsto(z, \zeta): z \in \partial \Omega_{+}^{\delta}, \xi=$ $z+\zeta \nu(z)$, which is well-defined if $\delta$ is small since $\partial \Omega \cap Q^{+}$is $C^{2}$. It can be shown that $J$ is continuous and $J(z, 0) \equiv 1$, therefore

$$
\lim _{\varepsilon \downarrow 0} L_{\varepsilon}\left(v_{\varepsilon}, A_{+}^{\delta}\right)=\int_{\partial \Omega_{+}^{\delta}} d \mathcal{H}^{1}(z)\left(\sigma_{c} \int_{-1}^{1} \cos ^{2}\left(\frac{\pi t}{2}\right) d t\right)=\sigma_{c} \mathcal{H}^{1}\left(\partial \Omega_{+}^{\delta}\right) .
$$


Equations (46), (47) and (48) yield

$$
\begin{aligned}
\limsup _{\varepsilon \downarrow 0} L\left(v_{\varepsilon}\right) & \leq\left(\sigma_{c} \wedge \sigma_{s}\right)\left(\mathcal{H}^{1}\left(\partial \Omega \backslash Q^{+}\right)-2 N \delta\right)+\sigma_{c} \mathcal{H}^{1}\left(\partial \Omega_{+}^{\delta}\right)+4 \sigma_{c} \mathcal{H}^{1}\left(C^{\delta}\right) \\
& \leq L(\Omega)-2 N\left(\sigma_{c} \wedge \sigma_{s}\right) \delta+4 \sigma_{c} \mathcal{H}^{1}\left(C^{\delta}\right)
\end{aligned}
$$

so that, sending $\delta$ to zero,

$$
\limsup _{\varepsilon \downarrow 0} L\left(v_{\varepsilon}\right) \leq L(\Omega) .
$$

Indeed, $\cap_{\delta>0} C^{\delta}=\bigcup_{k=1}^{N}\left\{a_{k}, b_{k}\right\}$ and the latter set has length zero, thus $\mathcal{H}^{1}\left(C^{\delta}\right)$ tends to zero.

We now build the associated functions $u_{\varepsilon}$. Let $c>0$ be the Lipschitz constant of $h$, we assume $c \geq 1$. If $\xi=(x, y) \in Q \backslash \Omega$, and $\xi^{\prime}=\left(x^{\prime}, h\left(x^{\prime}\right)\right) \in \partial \Omega, \mid h(x)-$ $h\left(x^{\prime}\right)|\leq c| x-x^{\prime} \mid$, so that $y-h(x) \leq c\left|x-x^{\prime}\right|+\left|h\left(x^{\prime}\right)-y\right|$, and we deduce that $y-h(x) \leq c \operatorname{dist}(\xi, \Omega)$. Thus, if in addition $v_{\varepsilon}(\xi)>0$, we have $\operatorname{dist}(\xi, \Omega)<2 \varepsilon$ so that $y-h(x)<2 c \varepsilon$, and $(x, y-2 c \varepsilon) \in \Omega$. We define for all $(x, y) \in Q$ the function $w_{\varepsilon}(x, y)=1 \wedge\left(v_{\varepsilon}(x, y-2 c \varepsilon) / v_{s}\right) \in H_{l o c}^{1}(Q): v_{\varepsilon}(x, y)>0$ implies $w_{\varepsilon}(x, y)=1$ (since $(x, y-2 c \varepsilon) \in \Omega$ and $v_{\varepsilon} \geq v_{s}$ in $\left.\Omega\right)$, and $w_{\varepsilon}(x, y)>0$ implies $(x, y-4 c \varepsilon) \in \Omega$.

We can define, for all $(x, y) \in \overline{Q^{+}}$,

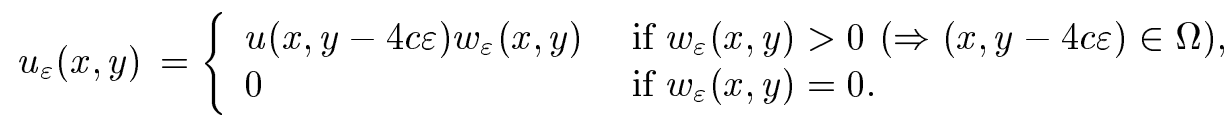

The function $u_{\varepsilon}(x, y)-(x, 0)$ is 1 -periodic in $x$ and vanishes for $y=0$, and $u_{\varepsilon}(x, y)=$ $u(x, y-4 c \varepsilon)$ as soon as $v_{\varepsilon}(x, y)>0$. We have

$$
e\left(u_{\varepsilon}\right)(x, y)=\left\{\begin{aligned}
e(u)(x, y-4 c \varepsilon) w_{\varepsilon}(x, y) & \\
+u(x, y-4 c \varepsilon) \odot \nabla w_{\varepsilon}(x, y) & \text { if } w_{\varepsilon}(x, y)>0 \\
0 & \text { if } w_{\varepsilon}(x, y)=0
\end{aligned}\right.
$$

where $a \odot b$ denotes the symmetrized tensor product, and $e\left(u_{\varepsilon}\right)(x, y)=e(u)(x, y-4 c \varepsilon)$ if $v_{\varepsilon}(x, y)>0$. Thus, for some constant $C>0$,

$$
\begin{aligned}
\int_{Q^{+}}\left(v_{\varepsilon}(\xi)\right. & \left.+\eta_{\varepsilon}\right) \mathbf{A} e\left(u_{\varepsilon}\right)(\xi): e\left(u_{\varepsilon}\right)(\xi) d \xi \\
\leq & \left(1+\eta_{\varepsilon}\right) \int_{\left\{v_{\varepsilon}>0\right\}} \mathbf{A} e\left(u_{\varepsilon}\right)(x, y-4 c \varepsilon): e\left(u_{\varepsilon}\right)(x, y-4 c \varepsilon) d x d y \\
& +C \eta_{\varepsilon} \int_{\left\{0<w_{\varepsilon}<1\right\}}|e(u)(x, y-4 c \varepsilon)|^{2}+|u(x, y-4 c \varepsilon)|^{2}\left|\nabla w_{e}(x, y)\right|^{2} d x d y \\
\leq & \left(1+\eta_{\varepsilon}\right) \int_{\Omega} \mathbf{A} e(u)(x, y): e(u)(x, y) d x d y \\
& +C \eta_{\varepsilon} \int_{\left\{0<v_{\varepsilon}<v_{s}\right\}}|e(u)(x, y-2 c \varepsilon)|^{2}+|u(x, y-2 c \varepsilon)|^{2}\left|\nabla v_{\varepsilon}(x, y) / v_{s}\right|^{2} d x d y
\end{aligned}
$$


We claim that this last integral goes to zero as $\varepsilon \downarrow 0$, so that

$$
\limsup _{\varepsilon \downarrow 0} \int_{Q^{+}}\left(v_{\varepsilon}(\xi)+\eta_{\varepsilon}\right) \mathbf{A} e\left(u_{\varepsilon}\right)(\xi): e\left(u_{\varepsilon}\right)(\xi) d \xi \leq \int_{\Omega} \mathbf{A} e(u)(\xi): e(u)(\xi) d \xi
$$

and in view of with (49), inequality (14) holds in this case.

Clearly, $\lim _{\varepsilon \downarrow 0} \eta_{\varepsilon} \int_{\left\{0<v_{\varepsilon}<v_{s}\right\}}|e(u)(x, y-2 c \varepsilon)|^{2} d x d y=0$. Then,

$$
\begin{aligned}
\eta_{\varepsilon} \int_{\left\{0<v_{\varepsilon}<v_{s}\right\}}|u(x, y-2 c \varepsilon)|^{2}\left|\nabla v_{\varepsilon}(x, y) / v_{s}\right|^{2} d x d y \\
\leq\|u\|_{\infty}^{2} \frac{\eta_{\varepsilon}}{v_{s}^{2} \varepsilon^{2}}\left\|\gamma^{\prime}\right\|_{\infty}^{2}\left|\left\{\xi \in Q^{+}: 0<v_{\varepsilon}(\xi)<v_{s}\right\}\right| \\
\quad \leq\left(\frac{\eta_{\varepsilon}}{\varepsilon}\right) \frac{\|u\|_{\infty}^{2}\left\|\gamma^{\prime}\right\|_{\infty}^{2}}{v_{s}^{2}} \frac{1}{\varepsilon}\left|\left\{\xi \in Q^{+}:\left|d_{\Omega}(\xi)\right|<2 \varepsilon\right\}\right|=o(1)
\end{aligned}
$$

since $\eta_{\varepsilon}=o(\varepsilon)$ and $\frac{1}{\varepsilon}\left|\left\{\left|d_{\Omega}\right|<2 \varepsilon\right\}\right|$ tends to $4 \mathcal{H}^{1}(\partial \Omega)$.

Therefore point (ii) of Theorem (2) is proved in the case where $\partial \Omega$ and $u$ have some regularity.

\subsubsection{Construction of $\left(v_{\varepsilon}, u_{\varepsilon}\right)$ in the general case}

Now, for an arbitrary $\Omega \in \mathcal{G}$ and $u$ in $X(\Omega)$, suppose we are able to build a sequence $\left(\Omega_{n}\right)_{n \geq 1}$ converging to $\Omega$ and $u_{n} \in X\left(\Omega_{n}\right) \cap L^{\infty}\left(\Omega_{n}\right)$ such that $u_{n} \rightarrow u$ in $L^{2}\left(A ; \mathbf{R}^{2}\right)$ for every $A \subset \subset \Omega$, each $\Omega_{n}$ is the sub-graph of a Lipschitz function, $\partial \Omega_{n} \cap Q^{+}$is $C^{2}$, $\partial \Omega_{n} \backslash Q^{+}$is a finite union of segments, and that satisfies

$$
\limsup _{n \rightarrow \infty} E\left(\Omega_{n}, u_{n}\right) \leq E(\Omega, u) .
$$

Then a simple diagonalization argument will lead to the result. Indeed, if we consider $\left(v_{n, \varepsilon}, u_{n, \varepsilon}\right)_{\varepsilon>0}$ obtained for each $n$ as described in section 6.2 .2 , we first build by induction a sequence $\varepsilon(n)$ such that for every $n, \varepsilon(n)<\min \{\varepsilon(n-1), 1 / n\}$, and such that

$$
\left\|u_{n, \varepsilon}-u_{n}\right\|_{L^{2}\left(\left\{\operatorname{dist}\left(\xi, Q \backslash \Omega_{n}\right)>1 / n\right\} ; \mathbf{R}^{2}\right)}<\frac{1}{n}
$$

and $v_{n, \varepsilon} \equiv 1$ on $\left\{\xi: \operatorname{dist}\left(\xi, Q \backslash \Omega_{n}\right)>1 / n\right\}$ as soon as $\varepsilon<\varepsilon(n)$, and

$$
\sup _{\varepsilon<\varepsilon(n)} E_{\varepsilon}\left(v_{n, \varepsilon}, u_{n, \varepsilon}\right) \leq E\left(u_{n}, \Omega_{n}\right)+\frac{1}{n} \text {. }
$$

Then, we let $u_{\varepsilon}=u_{n, \varepsilon}, v_{\varepsilon}=v_{n, \varepsilon}$ whenever $\varepsilon(n+1) \leq \varepsilon<\varepsilon(n)$. We have $v_{\varepsilon} \rightarrow \chi_{\Omega}$, $\limsup _{\varepsilon \downarrow 0} E\left(v_{\varepsilon}, u_{\varepsilon}\right) \leq E(u, \Omega)$, and if $A \subset \subset \Omega$, for large $n$ we have $A \subset\{\xi \in Q$ : $\left.\operatorname{dist}\left(\xi, Q \backslash \Omega_{n}\right)>1 / n\right\}$ so that for small $\varepsilon$,

$$
\begin{aligned}
\left\|u_{\varepsilon}-u\right\|_{L^{2}\left(A ; \mathbf{R}^{2}\right)} & \leq\left\|u_{\varepsilon}-u_{n(\varepsilon)}\right\|_{L^{2}\left(A ; \mathbf{R}^{2}\right)}+\left\|u_{n(\varepsilon)}-u\right\|_{L^{2}\left(A ; \mathbf{R}^{2}\right)} \\
& \leq 1 / n(\varepsilon)+\left\|u_{n(\varepsilon)}-u\right\|_{L^{2}\left(A ; \mathbf{R}^{2}\right)} \rightarrow 0
\end{aligned}
$$


as $\varepsilon \rightarrow 0$, where $n(\varepsilon)$ is defined by $\varepsilon(n(\varepsilon)+1) \leq \varepsilon<\varepsilon(n(\varepsilon))$ and goes to $+\infty$ as $\varepsilon \rightarrow 0$.

In order to build the sequence $\left(\Omega_{n}, u_{n}\right)$, we first assume we can find for every $n$ a $n$-Lipschitz function $h_{n} \leq h$, where $h$ satisfies $\Omega=\{(x, y) \in Q: y<h(x)\}$. This is proved in section $5.2\left(h_{n}\right.$ is given by equation $\left.(26)\right)$, and it is established that the sets $\Omega_{n}^{\prime}=\left\{(x, y) \in Q: y<h_{n}(x)\right\}$ converge to $\Omega$ and that $L\left(\Omega_{n}^{\prime}\right) \rightarrow L(\Omega)$. Now, if $\rho$ is a 1 -dimensional smoothing kernel $\left(\rho \in C^{\infty}(\mathbf{R}), \int_{\mathbf{R}} \rho=1, \rho \geq 1\right.$ and $\operatorname{supp} \rho \subset[-1 / 2,1 / 2])$, and $\rho_{n}(t)=n^{2} \rho\left(n^{2} t\right)$ for every $n \geq 1$, we let $g_{n}=h_{n} * \rho_{n}$ and $\Omega_{n}=\left\{(x, y) \in Q: y<g_{n}(x)\right\}$. It is not difficult to see that $\Omega_{n} \rightarrow \Omega$, that $\partial \Omega_{n}$ is smooth (in fact, $C^{\infty}$ ), and that $\partial \Omega_{n} \backslash Q^{+}$is a finite union of segments. We also have that $L\left(\Omega_{n}\right) \simeq L\left(\Omega_{n}^{\prime}\right) \rightarrow L(\Omega)$. Moreover, $g_{n}$ is Lipschitz $\left(g_{n} \in C^{\infty}\left(\mathbf{S}^{1}\right)\right)$ so that $\Omega_{n}$ is a Lipschitz sub-graph. Eventually it is easy to check that $g_{n} \leq h_{n}+c / n$ (where the constant $\left.c=\int_{\mathbf{R}}|t| \rho(t) d t\right)$, so that $\left\{(x, y-2 c / n):(x, y) \in \Omega_{n}\right\} \subset \subset \Omega_{n}^{\prime} \subseteq \Omega$ : therefore, we can build $u_{n} \in X\left(\Omega_{n}\right) \cap L^{\infty}\left(\Omega_{n}\right)$ as a suitable regularization of the function $u(x, y-2 c / n)$, which is defined in a neighborhood of $\Omega_{n}$.

\subsubsection{A remark on the integral of $v_{\varepsilon}$}

We now know how to build a family $\left(v_{\varepsilon}, u_{\varepsilon}\right)_{\varepsilon>0}$ for an arbitrary domain $\Omega \in \mathcal{G}$ and $u \in X(\Omega)$. In order to achieve the proof of Theorem 2 it remains to show that we can also impose that for every $\varepsilon, \int_{Q^{+}} v_{\varepsilon}(\xi) d \xi=|\Omega|$.

We have that $\int_{Q^{+}} v_{\varepsilon}(\xi) d \xi \rightarrow|\Omega|$ as $\varepsilon \downarrow 0$. Let now $\alpha_{\varepsilon}=\int_{Q^{+}} v_{\varepsilon}(\xi) d \xi /|\Omega|$, that converges to 1 . We define a new family $\left(v_{\varepsilon}^{\prime}, u_{\varepsilon}^{\prime}\right)_{\varepsilon>0}$ by

$$
\begin{gathered}
v_{\varepsilon}^{\prime}(x, y)=v_{\varepsilon}\left(x, \alpha_{\varepsilon} y\right), \\
\left(u_{\varepsilon}^{\prime x}(x, y), u_{\varepsilon}^{\prime y}(x, y)\right)=\left(u_{\varepsilon}^{x}\left(x, \alpha_{\varepsilon} y\right), \alpha_{\varepsilon} u_{\varepsilon}^{y}\left(x, \alpha_{\varepsilon} y\right)\right)
\end{gathered}
$$

for every $(x, y) \in Q^{+}$. It is not difficult to show that this family still satisfies point (ii) of Theorem 2, and that $\int_{Q^{+}} v_{\varepsilon}^{\prime}(\xi) d \xi=|\Omega|$ for every $\varepsilon>0$.

\subsubsection{A link between (i) and (ii) in Theorem 2}

The $\left(v_{\varepsilon}, u_{\varepsilon}\right)_{\varepsilon>0}$ constructed in section 6.2 have the property that the set $\Omega$ would be the set obtained from any subsequence $\left(v_{\varepsilon_{n}}\right)_{n \geq 1}$ by the method described in section 6.1. In particular, we deduce that, for this family $\left(v_{\varepsilon}, u_{\varepsilon}\right)$,

$$
\lim _{\varepsilon \downarrow 0} E_{\varepsilon}\left(v_{\varepsilon}, u_{\varepsilon}\right)=E(\Omega, u)
$$

\section{A Functions of bounded variation, Caccioppoli sets}

We review in this appendix some standard definitions and properties of the functions of bounded variation. For more details, the reader should consult $[11,7,10,2]$. 


\section{A.1 Definitions}

\section{A.1.1 Classical and measure theoretical total variation}

In the classical sense, the variation of a real-valued function $f: I \rightarrow \mathbf{R}$, defined on an interval $I \subseteq \mathbf{R}$, is given by

$$
\operatorname{Var}(f, I)=\sup \left\{\sum_{i=1}^{m}\left|f\left(t_{i}\right)-f\left(t_{i-1}\right)\right|: m \in \mathbf{N}, t_{0}, \ldots, t_{m} \in I, t_{0}<t_{1}<\cdots<t_{m}\right\} \text {. }
$$

The definition for a function $f: \mathbf{S}^{1} \rightarrow \mathbf{R}$ is similar. The function $f$ has bounded variation on $I$ in the classical sense if $\operatorname{Var}(f, I)<+\infty$.

In the measure theoretical sense, on the other hand, a function $f$ is said to belong to $B V(\Omega)$, the space of functions of bounded variation on the open domain $\Omega \subseteq \mathbf{R}^{N}$, $N \geq 1$, if and only if $f \in L^{1}\left(\mathbf{R}^{N}\right)$ and its distributional gradient $D f$ is a bounded vector measure on $\Omega$. In this case, the total variation of $f$ is the mass $|D f|(\Omega)$ of the measure $D f$. The definition is similar if $\Omega \subseteq \mathbf{S}^{1} \times \mathbf{R}$, which is usually the case in this paper.

\section{A.1.2 Sets with finite perimeter}

A Caccioppoli set, or set with finite perimeter in $\Omega$, is a set $E \subseteq \Omega$ such that the distributional gradient $D \chi_{E}$ of its characteristic function is a bounded vector measure on $\Omega$. (If $E$ is bounded we thus have $\chi_{E} \in B V(\Omega)$ ). In this definition, a set $E$ is of course identified with all sets $E^{\prime}$ such that $\left|E^{\prime} \triangle E\right|=0$. The perimeter of $E$ in $\Omega$ is the total variation $\left|D \chi_{E}\right|(\Omega)<+\infty$.

\section{A.2 Properties of functions with bounded variation}

\section{A.2.1 Links between both definitions}

If $I \subseteq \mathbf{R}$ (or $I \subseteq \mathbf{S}^{1}$ ) is an open interval, then

$$
\int_{I}|f(t)| d t<+\infty \text { and } \operatorname{Var}(f, I)<+\infty \Rightarrow f \in B V(I) .
$$

More precisely, the equivalence class of functions almost everywhere equal to $f$ is an element of $B V(I)$.

On the other hand, every $f \in B V(I)$ has a representative $g(g=f$ a.e. $)$ such that $\operatorname{Var}(g, I)=|D f|(I)<+\infty$. In fact, one can prove that

$$
|D f|(I)=\min \{\operatorname{Var}(g, I): g=f \text { a.e. }\} .
$$

Moreover, $f$ always have representatives $g^{\prime}$ with $\operatorname{Var}\left(g^{\prime}, I\right)=+\infty$ : for instance the characteristic function of $\mathbf{Q}$ belongs to $B V(\mathbf{R})$, but in the $B V$ sense $\chi_{\mathbf{Q}}=0$, whereas in the classical sense $\operatorname{Var}\left(\chi_{\mathbf{Q}}, \mathbf{R}\right)=2 \sharp \mathbf{Q}=+\infty$. 


\section{A.2.2 Continuity properties}

If $f \in B V_{l o c}(\Omega)$ we can define at each point $x \in \Omega$ the approximate lower limit of $f$ by

$$
f^{-}(x)=\sup \left\{t \in \mathbf{R}: \limsup _{\rho \downarrow 0} \frac{|\{f<t\} \cap B(x, \rho)|}{\rho^{N}}=0\right\}
$$

and the approximate upper limit $f^{+}(x)$ in the same way $\left(f^{+}(x)=-(-f)^{-}(x)\right)$. If $f^{+}(x)=f^{-}(x) \neq \pm \infty$ (this is true almost everywhere in $\Omega$ ), we set $\tilde{f}(x)=f^{+}(x)=$ $f^{-}(x)$ and say that the precise representative $\tilde{f}$ is approximately continuous at $x$.

If $N=1, \Omega=I \subseteq \mathbf{R}$ (or $I \subseteq \mathbf{S}^{1}$ ), and $f \in B V(I)$, for any $g$ representative of $f$ we have

$$
\operatorname{Var}(g, I)=|D f|(I) \Leftrightarrow g(x) \in\left[f^{-}(x), f^{+}(x)\right] \text { for every } x \in I .
$$

Moreover, in the 1-dimensional case, $f$ also has an approximate left limit at each point

$$
\begin{aligned}
f(x-0) & =\sup \left\{t \in \mathbf{R}: \limsup _{\rho \downarrow 0} \frac{|\{f<t\} \cap(x-\rho, x)|}{\rho}=0\right\} \\
& =\inf \left\{t \in \mathbf{R}: \limsup _{\rho \downarrow 0} \frac{|\{f>t\} \cap(x-\rho, x)|}{\rho}=0\right\},
\end{aligned}
$$

and a right limit $f(x+0)$ similarly defined. For every $x \in I,\{f(x-0), f(x+0)\}=$ $\left\{f^{-}(x), f^{+}(x)\right\}$. Notice that if $I=(a, b)$, then up to a constant $f(x-0)=|D f|((a, x))$ and $f(x+0)=|D f|((a, x])$. In particular, $f(x \pm 0)$ is continuous at each point of $I \backslash S_{f}$, where $S_{f}=\{f(x-0) \neq f(x+0)\}=\left\{f^{-}(x)<f^{+}(x)\right\}$ is the (at most countable) set of essential discontinuities of $f$. The representative $f^{-}$of $f$ is lower semi-continuous: it is the largest l.s.c. representative of $f$. Similarly, $f^{+}$is the lowest u.s.c. representative of $f$.

If $g$ is a representative of $f \in B V(I)$ such that $\operatorname{Var}(g, I)<+\infty$, at each $x_{0} \in I$ the (classical) left and right limits exist and we have

$$
\begin{aligned}
& \lim _{\substack{x \rightarrow x_{0} \\
x<x_{0}}} g(x)=g\left(x_{0}-0\right)=f\left(x_{0}-0\right), \text { and } \\
& \lim _{\substack{x \rightarrow x_{0} \\
x>x_{0}}} g(x)=g\left(x_{0}+0\right)=f\left(x_{0}+0\right) .
\end{aligned}
$$

\section{A.2.3 Sets and sub-graphs}

In the case where $f=\chi_{E}$, we let $E^{0}=\{\tilde{f}=0\}, E^{1}=\{\tilde{f}=1\}$, and define a reduced boundary by $\partial^{*} E=\Omega \backslash\left(E^{0} \cup E^{1}\right)$. It is known that the measure $D \chi_{E}$ is supported by the set $\partial^{*} E$, and that $\left|D \chi_{E}\right|=\mathcal{H}^{N-1}\left\llcorner\partial^{*} E\right.$, thus $\left|D \chi_{E}\right|(\Omega)=\mathcal{H}^{N-1}\left(\partial^{*} E\right)$ is the perimeter of $E$. 
If $I \subseteq \mathbf{R}$ (or $I \subseteq \mathbf{S}^{1}$ ), let $f \in B V(I)$. Then, the set $E=\{(x, y) \in I \times \mathbf{R}: y<$ $f(x)\} \subset I \times \mathbf{R}$ has finite perimeter. This can been shown for instance by approximating $f$ by a sequence of regular functions $f_{n}$ such that $\int_{I}\left|f_{n}^{\prime}(t)\right| d t \rightarrow|D f|(I)$, and invoking the lower semi-continuity of the total variation. We get

$$
\left|D \chi_{E}\right|(\Omega) \leq \liminf _{n \rightarrow \infty}\left|D \chi_{\left\{y<f_{n}(x)\right\}}\right|(\Omega)=\liminf _{n \rightarrow \infty} \int_{I} \sqrt{1+\left|f_{n}^{\prime}(t)\right|^{2}} d t \leq|I|+|D f|(I) .
$$

In fact, one can show that $\left|D_{x} \chi_{E}\right|(I)=|D f|(I)$ and $\left|D_{y} \chi_{E}\right|(I)=|I|$, so that the fact that the sub-graph $E$ has finite perimeter yields that $f \in B V(I)$, and

$$
|D f|(I) \leq \mathcal{H}^{1}\left(\partial^{*} E\right) \leq|I|+|D f|(I) .
$$

We define the open sets $A=\left\{y<f^{-}(x)\right\}$ and $B=\left\{y>f^{+}(x)\right\}$. It is not hard to see that $\bar{A}=B^{c}$ (and, equivalently, $\bar{B}=A^{c}$ ), so that $\stackrel{\circ}{A}=\bar{B}^{c}=\left(A^{c}\right)^{c}=A$, and $\partial A=\partial \bar{A}$. We can check easily from the definitions that $A \subset E^{1}$ and $B \subset E^{0}$, and in particular $\partial^{*} E \subseteq \partial A=\partial B=\left\{(x, y) \in I \times \mathbf{R}: f^{-}(x) \leq y \leq f^{+}(x)\right\}$. This last set can be shown to have finite length: in fact $\mathcal{H}^{1}(\partial A)=\mathcal{H}^{1}\left(\partial^{*} E\right)$. In particular, $A=B^{c}=E$ Lebesgue-essentially, and $\partial A=\partial^{*} E \mathcal{H}^{1}$-essentially.

One last result we want to state is a "classical" equivalent of (52). We claim that, if $g$ is a l.s.c. function, and if we consider the open set $\Omega=\{(x, y) \in I \times R: y<g(x)\}$, then

$$
\frac{1}{2} \operatorname{Var}(g, I) \leq \mathcal{H}^{1}(\partial \Omega) \leq|I|+\operatorname{Var}(g, I) .
$$

Indeed, if we consider $m \in \mathbf{N}$ and $t_{0}, \ldots, t_{m} \in I, t_{0}<t_{1}<\cdots<t_{m}$, then $\left|g\left(t_{i}\right)-g\left(t_{i-1}\right)\right| \leq \sqrt{\left|t_{i}-t_{i-1}\right|^{2}+\left|g\left(t_{i}\right)-g\left(t_{i-1}\right)\right|^{2}}$ which is the length of the segment joining $\left(t_{i-1}, g\left(t_{i-1}\right)\right)$ to $\left(t_{i}, g\left(t_{i}\right)\right)$. But $\partial \Omega \cap\left(\left[t_{i-1}, t_{i}\right] \times \mathbf{R}\right)$ contains at least a path connecting these to points, therefore this length is smaller than $\mathcal{H}^{1}\left(\partial \Omega \cap\left(\left[t_{i-1}, t_{i}\right] \times \mathbf{R}\right)\right)$. Summing over $i$ we get

$$
\sum_{i=1}^{m}\left|g\left(t_{i}\right)-g\left(t_{i-1}\right)\right| \leq \sum_{i=1}^{m} \mathcal{H}^{1}\left(\partial \Omega \cap\left(\left[t_{i-1}, t_{i}\right] \times \mathbf{R}\right)\right) \leq 2 \mathcal{H}^{1}(\partial \Omega),
$$

thus the left inequality in (53) holds.

To show the other inequality, we refer the reader to the techniques used in section 5 . Basically, one considers $\bar{g}$ such that $\stackrel{\circ}{\Omega}=\{(x, y) \in I \times \mathbf{R}, y<\bar{g}(x)\}$. One then can split $\mathcal{H}^{1}(\partial \Omega)=\mathcal{H}^{1}(\partial \bar{\Omega})+\mathcal{H}^{1}(\partial \Omega \backslash \partial \bar{\Omega})$. We get $\mathcal{H}^{1}(\partial \bar{\Omega}) \leq|I|+|D \bar{g}|(I)=|I|+\operatorname{Var}(\bar{g}, I)$ by (53), and we then show that $\partial \Omega \backslash \partial \bar{\Omega}=\bigcup_{\{g<\bar{g}\}}\{x\} \times[g(x), \bar{g}(x))$. We deduce that $2 \mathcal{H}^{1}(\partial \Omega \backslash \partial \bar{\Omega})=2 \sum_{\{g<\bar{g}\}}(\bar{g}(x)-g(x))=\operatorname{Var}(g, I)-\operatorname{Var}(\bar{g}, I)$, hence the result. 


\section{A.2.4 Co-area formula}

We eventually state the co-area formula: for every $f \in B V(\Omega)\left(\Omega \subseteq \mathbf{R}^{N}\right.$, or, as is common in this paper, $\left.\Omega \subseteq \mathbf{S}^{1} \times \mathbf{R}\right)$,

$$
|D f|(\Omega)=\int_{-\infty}^{+\infty}\left|D \chi_{\{f>t\}}\right|(\Omega) d t
$$

\section{References}

[1] G. Alberti. Variational models for phase transitions, an approach via $\Gamma-$ convergence. In G. Buttazzo et al, editor, Differential Equations and Calculus of Variations. Springer, 1999. to appear (available at http://cvgmt.sns.it/Preprints).

[2] L. Ambrosio, N. Fusco, and D. Pallara. Special Functions of Bounded Variation and Free Discontinuity Problems. Oxford University Press, Oxford, 2000. (to appear).

[3] L. Ambrosio and V. M. Tortorelli. Approximation of functionals depending on jumps by elliptic functionals via $\Gamma$-convergence. Comm. Pure Appl. Math., 43:999$1036,1990$.

[4] E. Bonnetier, R. S. Falk, and M. A. Grinfeld. Analysis of a one-dimensional variational model of the equilibrium shape of a deformable crystal. M2AN Math. Model. Numer. Anal., 33(3):573-591, 1999.

[5] J. W. Cahn and J. E. Hilliard. Free energy of a nonuniform system I - interfacial free energy. J. Chem. Phys., 28:258-267, 1958.

[6] G. Dal Maso. An introduction to $\Gamma$-convergence. Progress in Nonlinear Differential Equations and their Applications. Birkhäuser, Boston, 1993.

[7] L. C. Evans and R. F. Gariepy. Measure Theory and Fine Properties of Functions. CRC Press, Boca Raton, 1992.

[8] K. J. Falconer. The geometry of fractal sets. Cambridge University Press, Cambridge, 1985.

[9] R.S. Falk. Personal communication.

[10] H. Federer. Geometric Measure Theory. Classics in Mathematics. Springer, 1969.

[11] E. Giusti. Minimal surfaces and functions of bounded variation. Birkhäuser, Boston, 1984. 
[12] S. Golab. Sur quelques points de la théorie de la longueur. Annales de la Société Polonaise de Mathématiques, VII:227-241, 1928. (Rocznik polskiego tow. matematycznego).

[13] M. A. Grinfeld. Stress driven instabilities in crystals: mathematical models and physical manifestations. J. Nonlinear Sci., 3:35-83, 1993.

[14] P. H. Leo, J. S. Lowengrub, and H. J. Jou. A diffusive interface model for microstructural evolution in elastically stressed solids. Acta mater, 46(6):2113-2130, 1998.

[15] L. Modica and S. Mortola. Il limite nella $\Gamma$-convergenza di una famiglia di funzionali ellittici. Boll. Un. Mat. Ital. A (5), 14-A:526-529, 1977.

[16] J.-M. Morel and S. Solimini. Variational Methods in Image Segmentation. Birkhäuser, Boston, 1995.

[17] MSR bulletin, April 1996.

[18] J. Muller and M. Grant. Model of surface instabilities induced by stress. Phys. Rev. Lett., 82:1736-1739, 1999.

[19] R. H. Nochetto, S. Rovida, M. Paolini, and C. Verdi. Variational approximation of the geometric motion of fronts. In Motion by mean curvature and related topics (Trento, 1992), pages 124-149, Berlin, 1994. de Gruyter.

[20] M. Paolini and C. Verdi. Asymptotic and numerical analyses of the mean curvature flow with a space-dependent relaxation parameter. Asymptotic Analysis, 5:553$574,1992$. 